\title{
EFT anomalous dimensions from the S-matrix
}

\author{
Joan Elias Miró, ${ }^{a}$ James Ingoldby $^{a}$ and Marc Riembau ${ }^{b}$ \\ ${ }^{a}$ Abdus Salam International Centre for Theoretical Physics, \\ Strada Costiera 11, 34151, Trieste, Italy \\ ${ }^{b}$ Université de Genève, \\ Ernest-Ansermet 24, 1211 Genève, Switzerland \\ E-mail: eliasmiro@ictp.it, ingoldby@ictp.it, marc.riembau@unige.ch
}

\begin{abstract}
We use the on-shell S-matrix and form factors to compute anomalous dimensions of higher dimension operators in the Standard Model Effective Field Theory. We find that in many instances, these computations are made simple by using the on-shell method. We first compute contributions to anomalous dimensions of operators at dimension-six that arise at one-loop. Then we calculate two-loop anomalous dimensions for which the corresponding one-loop contribution is absent, using this powerful method.
\end{abstract}

Keywords: Beyond Standard Model, Effective Field Theories, Renormalization Group, Scattering Amplitudes

ARXIV EPRINT: 2005.06983 


\section{Contents}

1 Introduction 1

2 One-loop RGEs of dimension-six operators 4

2.1 Contributions at order $\lambda \quad 5$

2.1.1 $\partial^{2} H^{4}$ operators $\quad 5$

$\begin{array}{ll}\text { 2.1.2 The rest of the order } \lambda \text { corrections } & 7\end{array}$

2.2 Contributions from gauge interactions 9

2.2.1 IR divergences $\quad 9$

$\begin{array}{ll}2.2 .2 \text { Gauge mixing } & 11\end{array}$

$\begin{array}{lll}2.3 & \text { Non-minimal form factors } & 12\end{array}$

3 Structure of the anomalous dimension matrix 14

4 The 'easy' two-loop anomalous dimensions $\quad 16$

$4.1\langle\cdot\rangle^{2} \rightarrow\langle\cdot\rangle^{3}:$ 4-point to 3-point 17

$4.2\langle\cdot\rangle \rightarrow\langle\cdot\rangle^{2}:$ 5-point to 4-point $\quad 19$

$4.31 \rightarrow\langle\cdot\rangle: 6$-point to 5-point 21

5 Conclusions $\quad 22$

A Phase space integrals $\quad 23$

A.1 The $2 \rightarrow 2$ case 25

A.2 The $3 \rightarrow 2$ case 25

$\begin{array}{ll}\text { B Amplitudes } & 27\end{array}$

\section{Introduction}

The discovery of the Higgs particle [1, 2], Higgs coupling measurements, and a plethora of beyond the Standard Model (SM) searches suggest that there is an energy gap between the electroweak scale and any new physics scale. Physics within this energy gap is appropriately described by the SM, together with a tower of operators encoding the deformations generated by the new dynamics. Given such separation of scales, the Renormalisation Group (RG) mixing of higher-dimension SM Effective Field Theory (EFT) operators can lead to important physical effects on precision observables. The RG running of SM EFT operators down from a hypothetical new physics scale to the EW scale is governed by the operator anomalous dimensions.

The anomalous dimension of an operator can be computed using a number of different methods. For instance, the S-matrix elements, the effective action, or form factors, are 
subject to a Callan-Symanzik equation, a.k.a. renormalisation group equation (RGE), that depends on the anomalous dimensions. In this work we will compute the anomalous dimensions of SM dimension-six operators through the RGE's satisfied by the form factors.

The form factors (FFs) are defined by

$$
F_{O}(\vec{n}) \equiv \text { out }\langle\vec{n}|O(0)| 0\rangle,
$$

where $O(x)$ is a local and gauge invariant operator and out $\langle\vec{n}|$ is a multi-particle asymptotic state. These are the same states that are used to define the scattering $S$-matrix elements

$$
S_{n m} \equiv\langle\vec{n}|\hat{S}| \vec{m}\rangle={ }_{\text {out }}\langle\vec{n} \mid \vec{m}\rangle_{\text {in }} .
$$

In perturbation theory, the calculation of the FFs involves regularising IR and UV divergences. For definiteness we will be using dimensional regularisation and the $\overline{\mathrm{MS}}$ scheme so that the FF computed at a fixed order in perturbation theory will depend on the 't Hooft scale $\mu$. However, it satisfies the Callan-Symanzik equation

$$
\left(\mu \partial_{\mu}+\gamma-\gamma_{\mathrm{IR}}+\beta_{g} \partial_{g}\right) F_{O}(\vec{n} ; \mu)=0,
$$

where $\gamma$ is the anomalous dimension matrix, $\gamma_{\mathrm{IR}}$ is the IR anomalous dimension, and $\beta_{g}$ denotes collectively the beta function of the couplings in the theory. $\gamma_{\mathrm{IR}}$ is diagonal in the space of kinematically independent operators and it is due to soft and/or collinear emission of particles.

Next we derive a relation between the form-factors and the S-matrix. We need three ingredients:

1) Unitarity $F_{O}(\vec{n}) \equiv$ out $\langle\vec{n}|O(0)| 0\rangle=\sum_{\vec{m} \text { out }}\langle\vec{n} \mid \vec{m}\rangle_{\text {in in }}\langle\vec{m}|O(0)| 0\rangle$.

2) The CPT theorem implies out $\langle\vec{n}|O(0)| 0\rangle=\left\langle 0\left|O^{\dagger}(0)\right| \vec{n}\right\rangle_{\text {in }}$.

3) Analyticity $F_{O}^{*}\left(s_{i j}-i \epsilon\right)=F_{O}\left(s_{i j}+i \epsilon\right)$.

Regarding 2), recall that CPT is a symmetry transformation that is represented on the Hilbert space by an anti-linear and anti-unitary operator. Thus it relates in/out states when inserted in the inner product, while acts on local operators as $\mathrm{CPT} O(x)[\mathrm{CPT}]^{-1}=$ $O^{\dagger}(-x)$. Thus, point 2) follows from the statement out $\langle\vec{n}|O(0)| 0\rangle$ $=\left\langle 0\left|[\mathrm{CPT}]^{-1} \mathrm{CPT}^{\dagger}(0)[\mathrm{CPT}]^{-1} \mathrm{CPT}\right| \vec{n}\right\rangle_{\text {in }}$.

As for 3), note that due to Lorentz invariance $F_{O}\left(\vec{n}\left(s_{i j}+i \epsilon\right)\right) \equiv F_{O}\left(s_{i j}+i \epsilon\right)$ must depend on the Mandelstam invariants $s_{i j}=2 p_{i} \cdot p_{j}$, for $i, j=1, \ldots n$, which we collectively denote by $F_{O}\left(s_{i j}+i \epsilon\right)$, where $\epsilon>0$ is a small positive parameter arising from the Feynman $i \epsilon$-prescription. The real analyticity relation in 3$)$ is definitely true in perturbation theory because complex conjugation of $F_{O}$ amounts to complex conjugating the time-ordered propagators into anti-time-ordered propagators and thus $s_{i j}+i \epsilon \rightarrow s_{i j}-i \epsilon$. This full counter-clockwise rotation in the $s_{i j}$ complex plane can be generated by a complex dilatation of the momenta. Indeed, defining the momentum space dilatation generator

$$
D \equiv \sum_{\text {all particles }} p_{i} \frac{\partial}{\partial p_{i}},
$$


the relation in 3) can be written as $e^{-i \pi D} F_{O}^{*}\left(s_{i j}+i \epsilon\right)=F_{O}\left(s_{i j}+i \epsilon\right)$, which together with 1) and 2) implies

$$
e^{-i \pi D} F_{O}^{*}(\vec{n})=\sum_{\vec{m}} S_{n m} F_{O}^{*}(\vec{m}) .
$$

This equation was derived in [3] using a version of the optical theorem. The derivation of (1.5) that we present here though is similar to the proof of the two-dimensional generalised Watson theorem [4]. ${ }^{1}$ In two-dimensions complex conjugation in the rapidity plane $\theta$ acts as $F_{O}^{*}(\theta)=F_{O}(-\theta)$, where $\theta=\left|\theta_{1}-\theta_{2}\right|$ is the rapidity difference of two incoming particles of momenta $\left\{p_{1}, p_{2}\right\}$, and $\left(p_{1}+p_{2}\right)^{2}=4 m^{2} \cosh ^{2}\left(\theta_{1}-\theta_{2}\right) / 2$. Then, by requiring only the extra assumption of a factorised two-dimensional S-matrix, equation (1.5) for $n=2$ reduces to the generalised Watson theorem $F_{O}(\theta)=F_{O}(-\theta) S(\theta)$ [4], where $S(\theta)$ denotes the $2 \rightarrow 2$ scattering element and we are omitting flavour indices; this equation is exactly satisfied by many solvable two-dimensional QFTs.

For the purposes of computing UV anomalous dimensions, one can consider all particles as massless. Next, following the observation of [3], we relate the Callan-Symanzik equation (1.3) to the action of the momentum dilatation operator

$$
D F_{O} \approx-\mu \partial_{\mu} F_{O}^{(1)}=\left(\gamma-\gamma_{\mathrm{IR}}+\beta_{g} \partial_{g}\right)^{(1)} F_{O}^{(0)} .
$$

Indeed, in dimensional regularisation there is a single renormalisation scale $\mu$ and we can trade derivatives of $\sum_{i} p_{i} \partial_{p_{i}}$ by $-\mu \partial_{\mu}$. Thus, using (1.5), (1.6) and expanding in powers of $D$ at first non-trivial order one gets [3]

$$
\left\langle\vec{n}\left|O_{j}\right| 0\right\rangle^{(0)}\left(\gamma_{j i}-\gamma_{\mathrm{IR}}^{i} \delta_{i j}\right)^{(1)}=-\frac{1}{\pi}\left\langle\vec{n}\left|\mathcal{M} \otimes O_{i}\right| 0\right\rangle^{(0)},
$$

where as usual we have separated the S-matrix as $S=\mathbb{1}+i \mathcal{M}$. The first factor on the left $\left\langle\vec{n}\left|O_{j}\right| 0\right\rangle^{(0)}$ is a minimal form factor, namely the lowest order FF such that does not vanish in the free theory limit. The minimal FF is a polynomial in the kinematic variables: the momenta and polarisations of the particles. The action of $\beta_{g} \partial_{g}$ can be neglected because $\left\langle\vec{n}\left|O_{j}\right| 0\right\rangle^{(0)}$ does not depend on the couplings of the theory. On the right hand side, ' $\otimes$ ' denotes an insertion of $\mathbb{1}=\sum_{\vec{m}}|\vec{m}\rangle_{\text {in in }}\langle\vec{m}|$ as in 2). The $\mathcal{M}$-operator and the form factor on the right are consistently computed in perturbation theory so that it leads to a polynomial function like the l.h.s. minimal FF. For instance, note that if the leading single $\log \mu$ in the $\mathrm{FF}$ of the l.h.s. in (1.5) arises at $\ell$-loops, then applying $-\mu \partial_{\mu}$ as in (1.6) extracts the anomalous dimension at $\ell$-loop order. Thus '(1)' in (1.7) indicates the coefficient of the leading single $\log \mu$, which typically (but not necessarily) arises at one-loop. From here on we will neglect the super-indices $(\cdots)^{(n)}, n=0,1$.

In section 2 we will discuss the method and compute representative one-loop anomalous dimensions of the SM dimension-six operators, and compare the results with previous literature. In section 3 we begin by summarising the pattern of vanishing entries in the matrix of one-loop anomalous dimensions. This allows us to identify those two-loop anomalous dimensions that are most interesting, namely those two-loop RG mixings that vanish at

\footnotetext{
${ }^{1}$ Watson's theorem [5, 6] is e.g. reviewed in [7], formulated as a relation between components of the S-matrix.
} 
one-loop. These can be efficiently obtained with the on-shell methodology and we compute two-loop anomalous dimensions of this type in section 4 . Finally we summarise our results and conclude in section 5 .

\section{One-loop RGEs of dimension-six operators}

In this section we apply (1.7) in a series of examples of one-loop RGE mixing of the SM dimension-six operators. The one loop contributions to the right hand side of (1.7) involve a tree-level form factor and a tree-level S-matrix element, that are contracted with a twoparticle phase space integral. Note that the scattering matrix in the right hand side of (1.5) is not the fully connected S-matrix. Thus we should also consider contributions that involve a scattering matrix with disconnected pieces

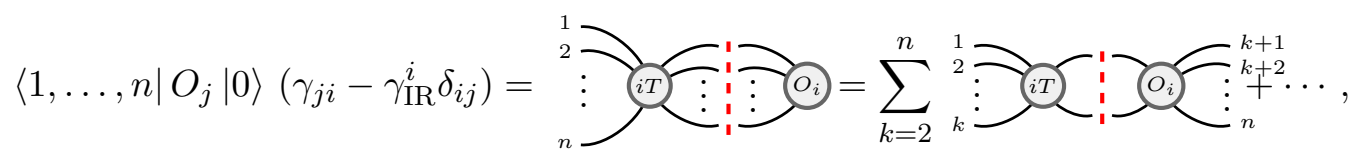

where the lines to the right of the last FF are the disconnected particles of the $S$-matrix. ${ }^{2}$ The dots in (2.1) denote the sum of any possible permutation of the external particles, and higher loop contributions.

For the purposes of computing the UV anomalous dimensions, one can consider all SM particles as massless, or equivalently consider the SM in the symmetric phase of $\mathrm{SU}(2)_{L}$. Then, since all the four-momenta involved in the problem $(2.1)$ is null $p^{2}=0$, it is convenient to use spinor helicity formalism [8-10]. An on-shell massless spin $s$ particle is characterised by its helicity $h= \pm|s|$ and a pair of spinors $\left\{\lambda_{a}, \tilde{\lambda}_{\dot{a}}\right\}$. The spinors $\left\{\lambda_{a}, \tilde{\lambda}_{\dot{a}}\right\}$ transform in the representations $(1 / 2,0)$ and $(0,1 / 2)$ of the universal cover of the Lorentz group $\operatorname{SL}(2, \mathbb{C})$, respectively. The basic invariant tensors are the antisymmetric Levi-Civita $\epsilon^{a b}, \epsilon^{\dot{a} \dot{b}}$, and $\sigma^{\mu}=\left(1, \sigma^{i}\right)$ with $\sigma^{i}$ the Pauli matrices. Given two particles with spinors $\left\{\left(\lambda_{i}\right)_{a},\left(\tilde{\lambda_{i}}\right)_{\dot{a}}\right\}$ where $i=1,2$, two simple Lorentz invariants are

$$
\left(\lambda_{1}\right)_{a}\left(\lambda_{2}\right)_{b} \epsilon^{a b} \equiv\langle 12\rangle \quad \text { and } \quad\left(\tilde{\lambda}_{1}\right)_{\dot{a}}\left(\tilde{\lambda}_{2}\right)_{\dot{b}} \epsilon^{\dot{a} \dot{b}} \equiv[12],
$$

where we have introduced the commonly used angle and square bracket notation. Another basic invariant is $\left(p_{i}+p_{j}\right)^{2}=\langle i j\rangle[j i] \equiv s_{i j}$, where we are using mostly minus $\eta=(+---)$ metric signature. Our convention is $p^{\mu}=\lambda^{a}\left(\sigma^{\mu}\right)_{a \dot{a}} \tilde{\lambda}^{\dot{a}}$, and as usual we consider $\lambda$ and $\tilde{\lambda}$ as independent complex spinors (i.e. two copies of $\operatorname{SL}(2, \mathbb{C})$ ), however for real physical momenta (with positive energy) we should identify $\tilde{\lambda}=+\lambda^{*}$. See for instance $[11,12]$ for further details on the spinor helicity formalism and on-shell scattering amplitudes techniques.

As usual, we factor out a Dirac delta of total momenta conservation from the interacting and connected $i \mathcal{M}$ matrix

$$
\left\langle 12 \ldots k|i \mathcal{M}| 1^{\prime} 2^{\prime} \ldots k^{\prime}\right\rangle=(2 \pi)^{4} \delta^{(4)}\left(\sum_{i=1}^{k} p_{i}-\sum_{i=1}^{k^{\prime}} p_{i}^{\prime}\right) i M\left(1 \ldots k ; 1 \ldots k^{\prime}\right),
$$

\footnotetext{
${ }^{2}$ Indeed, from $\int \frac{d \mathrm{LI}^{\prime}}{2 \pi}\left\langle 1, \ldots, n|i \mathcal{M}| 1^{\prime}, 2^{\prime}, 3^{\prime}, \ldots, k^{\prime}\right\rangle\left\langle 1^{\prime}, 2^{\prime}, 3^{\prime}, \ldots, k^{\prime}\left|O_{i}\right| 0\right\rangle$ pick a connected $2 \rightarrow k$ scattering element to reduce it to $\int \frac{d \mathrm{LI}^{\prime}}{2 \pi}\left\langle 1, \ldots, k|i \mathcal{M}| 1^{\prime}, 2^{\prime}\right\rangle\left\langle 1^{\prime}, 2^{\prime}, k+1, \ldots, n\left|O_{i}\right| 0\right\rangle$. The particles to the right of the last FF in (2.1) may involve tree-level interactions of the disconnected S-matrix, but we have absorbed those into the FF, such that both the S-matrix and the FF are connected.
} 
where $M$ is a function of the spinor variables $\left\{\lambda_{i_{a}}, \tilde{\lambda}_{i_{\dot{a}}}\right\}$ with $i \in\{1, \ldots k\}$ and $\left\{\lambda_{i_{a}}^{\prime}, \tilde{\lambda}_{i_{\dot{a}}}^{\prime}\right\}$ with $i \in\left\{1, \ldots k^{\prime}\right\}$.

Starting with simplest things first, below we will show various one-loop computations that involve only the $2 \rightarrow 2$ matrix elements in first term of the r.h.s. of (2.1). Thus we need to perform the following type of integrals

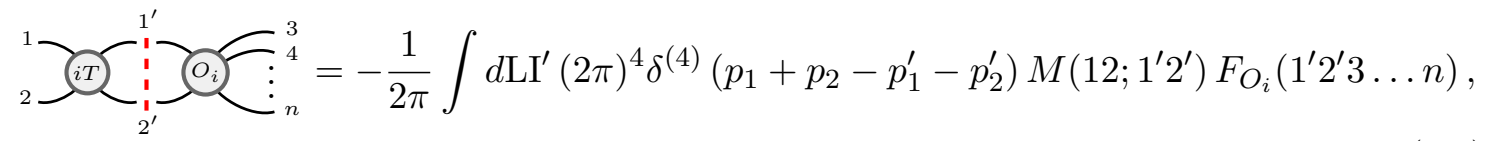

where from here on we leave implicit the sum over all permutations of the external particles, and $d \mathrm{LI}^{\prime}$ is the Lorentz invariant integral measure over all the $p_{i}^{\prime}$ variables, in this case $d \mathrm{LI}^{\prime}=\prod_{i=1}^{2} \frac{d^{3} p_{i}^{\prime}}{(2 \pi)^{3} 2 p_{i}^{\prime}}$. The integral in (2.4) can be neatly performed sticking to the spinor formalism for the FF and the $M$ function. Since $p_{1}+p_{2}=p_{1}^{\prime}+p_{2}^{\prime}$ throughout the whole phase-space integration domain, it is useful to parametrise the $\lambda_{i}^{\prime}$ s in terms of the external particle's spinors by $[3,13]$

$$
\left(\begin{array}{l}
\lambda_{1}^{\prime} \\
\lambda_{2}^{\prime}
\end{array}\right)=\left(\begin{array}{cc}
\cos \theta & -\sin \theta e^{i \phi} \\
\sin \theta e^{-i \phi} & \cos \theta
\end{array}\right)\left(\begin{array}{l}
\lambda_{1} \\
\lambda_{2}
\end{array}\right)
$$

and similarly for the $\tilde{\lambda}$ 's spinors. Then, the two-particle phase-space integral becomes an integral over the rotation angles $\{\theta, \phi\}$ only, and (2.4) reduces to

$$
F_{O_{j}}(12 \ldots n)\left(\gamma_{j i}-\gamma_{\mathrm{IR}}^{i} \delta_{i j}\right)=\frac{-1}{16 \pi^{2}} \int_{0}^{2 \pi} \frac{d \phi}{2 \pi} \int_{0}^{\pi / 2} 2 \sin \theta \cos \theta d \theta M\left(1,2 ; 1^{\prime} 2^{\prime}\right) F_{O_{i}}\left(1^{\prime} 2^{\prime} 3 \ldots n\right) .
$$

For future reference we define the angular integration measure $d \Omega_{2} \equiv \frac{d \phi}{2 \pi} 2 \cos \theta \sin \theta d \theta$. See appendix A for further details.

\subsection{Contributions at order $\lambda$}

In the following we compute the anomalous dimensions of the SM dimension-six operators at order $\lambda$ with the presented on-shell formalism. In this case $\gamma_{I R}$ is absent since there are no collinear or soft divergences and thus using (2.6) we can directly compute the UV anomalous dimensions.

The tree-level $M$-matrix amplitude at $O(\lambda)$ due to $\delta \mathcal{L}=-\lambda|H|^{4}$ is given by

$$
M\left(1_{i} 2_{j} 3_{k}^{*} 4_{l}^{*}\right)=-2 \lambda\left(\delta_{i}^{k} \delta_{j}^{l}+\delta_{j}^{k} \delta_{i}^{l}\right),
$$

where we have taken all the particles as outgoing and the indices run over the components of the Higgs doublet $H$. By crossing outgoing particles into ingoing we generate the $2 \rightarrow 2$ amplitudes $M\left(1_{i} 2_{j} ; 1_{k} 2_{l}\right), M\left(1_{i} 2_{j}^{*} ; 1_{k} 2_{l}^{*}\right)$, etc.

\subsection{1 $\partial^{2} H^{4}$ operators}

We need to specify the FFs that overlap with the scattering element in (2.7). Starting with operators involving four Higgses, there are two independent dimension-six operators 
$O_{\|}=|H|^{2}\left|D^{\mu} H\right|^{2}$ and $O_{\perp}=\left|H^{\dagger} D_{\mu} H\right|^{2}$. Their minimal FFs with a four particle state are

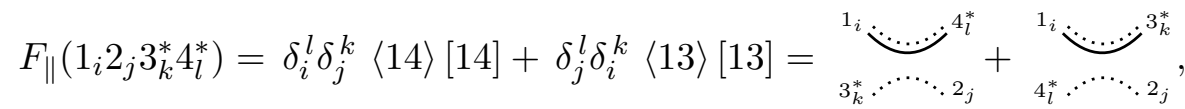

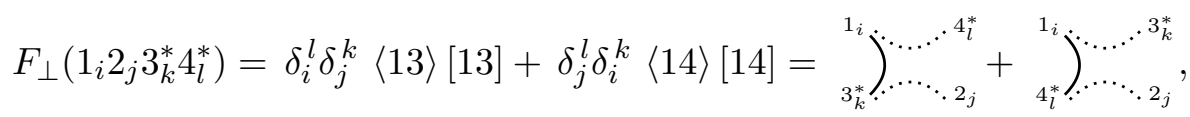

where we have used momentum conservation $\sum_{i=1}^{4} p_{i}=0 .{ }^{3}$ The two operators are in correspondence with the two possible ways to contract the momenta (solid line in (2.8), (2.9)) of two particles with two antiparticles that carry a flavour index (dotted line).

Next we need to connect the FFs in (2.8), (2.9) with the matrix element (2.7) in the various possible ways or channels

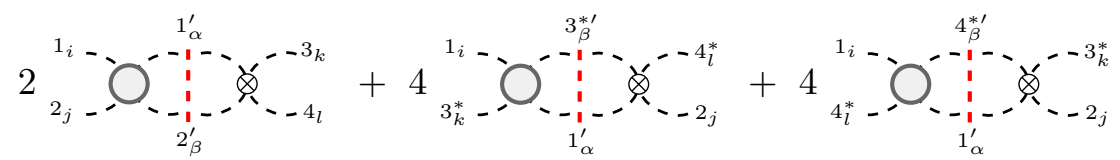

where from here on we represent on-shell scalar particle lines with a dashed line, a crossed dot denotes an insertion of the operator and a grey blob the $\mathcal{M}$-matrix. Regarding the multiplicative factor in each channel, note that a factor of two is due to crossing, ${ }^{4}$ while the last two channels get and extra factor of two because $\left|H_{a}, H_{b}^{*}\right\rangle$ and $\left|H_{b}, H_{a}^{*}\right\rangle$ are distinct states.

With this it is straightforward to compute the various channels. Starting with the $F_{\perp}$ form factor, the first channel gives

$$
-\frac{1}{16 \pi^{2}} \int d \Omega_{2}\left[-2 \lambda\left(\delta_{i}^{\alpha} \delta_{j}^{\beta}+\delta_{j}^{\alpha} \delta_{i}^{\beta}\right)\right]\left[\delta_{\alpha}^{l} \delta_{\beta}^{k}\left\langle 1^{\prime} 3\right\rangle\left[1^{\prime} 3\right]+\delta_{\beta}^{l} \delta_{\alpha}^{k}\left\langle 1^{\prime} 4\right\rangle\left[1^{\prime} 4\right]\right],
$$

where $d \Omega_{2}$ is the angular integration in (2.6). The spinor $\left\langle 1^{\prime}\right|$ is rotated according to (2.5), which gives $\left\langle 1^{\prime} i\right\rangle=\cos \theta\langle 1 i\rangle-\sin \theta e^{i \phi}\langle 2 i\rangle$. The $\phi$ integral sets to zero the terms with a phase $e^{i \phi}$, thus $\int d \Omega_{2}\left\langle 1^{\prime} i\right\rangle\left[1^{\prime} i\right]=\int d \Omega_{2}\left(\cos ^{2} \theta\langle 1 i\rangle[1 i]+\sin ^{2} \theta\langle 2 i\rangle[2 i]\right)$ for $i=3$, 4. After doing the $\theta$ integral we get

$$
(2.11)=\frac{2 \lambda}{16 \pi^{2}}\left[\delta_{i}^{l} \delta_{j}^{k}+\delta_{j}^{l} \delta_{i}^{k}\right][\langle 13\rangle[13]+\langle 14\rangle[14]] .
$$

The second and third channels in (2.10) are done in the same way. The second one gives

$$
-\frac{1}{16 \pi^{2}} \int d \Omega_{2} M\left(1_{i} 3_{k}^{*} ; 1_{\alpha}^{\prime} 3_{\beta}^{* \prime}\right) F_{\perp}\left(1_{\alpha}^{\prime} 2{ }_{j} 3_{\beta}^{* \prime} 4_{l}^{*}\right)=-\frac{\lambda\langle 13\rangle[13]}{16 \pi^{2}}\left[\delta_{i}^{k} \delta_{j}^{l}(N-1)-2 \delta_{i}^{l} \delta_{j}^{k}\right],
$$

where $N=2$ is the dimension of the fundamental representation of the Higgs doublet. In this case we rotate $\left(\left|1^{\prime}\right\rangle,\left|3^{\prime}\right\rangle\right)=R(\theta, \phi) .(|1\rangle,|3\rangle)$. Finally the third channel

$$
-\frac{1}{16 \pi^{2}} \int d \Omega_{2} M\left(1_{i} 4_{l}^{*} ; 1_{\alpha}^{\prime} 4_{\beta}^{* \prime}\right) F_{\perp}\left(1_{\alpha}^{\prime} 2_{j} 3_{k} 4_{\beta}^{* \prime}\right)=-\frac{\lambda\langle 14\rangle[14]}{16 \pi^{2}}\left[\delta_{i}^{l} \delta_{j}^{k}(N-1)-2 \delta_{i}^{k} \delta_{j}^{l}\right]
$$

\footnotetext{
${ }^{3} \mathrm{FFs}$ in momentum space, $F_{O}\left(p_{1} \ldots p_{n} ; q\right) \equiv \int d^{4} x e^{i q x} F_{O}\left(p_{1}, \ldots, p_{n}\right)$, have the support of the fourmomentum conservation delta function $\delta^{(4)}\left(q+\Sigma_{i} p_{i}\right)$. We will indistinguishably refer to $F_{O}\left(p_{1} \ldots p_{n} ; 0\right)$ by $F_{O}\left(p_{1} \ldots p_{n}\right)$.

${ }^{4}$ I.e. one gets the same result when exchanging $\{1,2\} \leftrightarrow\{3,4\},\{1,3\} \leftrightarrow\{2,4\}$ and $\{1,4\} \leftrightarrow\{2,3\}$ in the first second and third channel respectively.
} 
can be obtained from the second channel upon exchanging $3 \leftrightarrow 4$ and $k \leftrightarrow l$ on (2.13). After adding all the contributions, $2 \cdot(2.12)+4 \cdot(2.13)+4 \cdot(2.14)$, we get

$$
\frac{4 \lambda}{16 \pi^{2}}\left[3 F_{\perp}\left(1_{i} 2_{j} 3_{k}^{*} 4_{l}^{*}\right)+(2-N) F_{\|}\left(1_{i} 2_{j} 3_{k}^{*} 4_{l}^{*}\right)\right] .
$$

Thus for a SM Higgs doublet, we have that $N=2$ and the renormalisation is diagonal.

Proceeding in a similar fashion for the $F_{\|}$form factor we get the following result for the various channels: $t_{1}=2 \kappa\left[\delta_{i}^{l} \delta_{j}^{k}+\delta_{i}^{k} \delta_{j}^{l}\right]\langle 12\rangle[12], t_{2}=-\kappa\left[\delta_{i}^{k} \delta_{j}^{l}(2 N+1)+\delta_{i}^{l} \delta_{j}^{k}\right]\langle 13\rangle[13]$ and $t_{3}=-\kappa\left[\delta_{j}^{k} \delta_{i}^{l}(2 N+1)+\delta_{j}^{l} \delta_{i}^{k}\right]\langle 14\rangle[14]$, where $\kappa \equiv-2 \lambda\left(16 \pi^{2}\right)^{-1}$. Adding the different channels, $2 t_{1}+4 t_{2}+4 t_{3}$, gives

$$
\frac{8 \lambda}{16 \pi^{2}}(2 N-1) F_{\|}\left(1_{i} 2_{j} 3_{k}^{*} 4_{l}^{*}\right)
$$

From (2.15) and (2.16) we read the order $\lambda$ anomalous dimension matrix of the $\partial^{2} H^{4}$-type SM operators $\gamma_{c_{\perp}}=12 \lambda \gamma_{c_{\perp}} / 16 \pi^{2}$ and $\gamma_{c_{\|}}=24 \lambda \gamma_{c_{\|}} / 16 \pi^{2}$, in agreement with [14-16]. ${ }^{5}$

\subsubsection{The rest of the order $\lambda$ corrections}

After having set the notation and basic steps, it is straightforward to compute the rest of the order $\lambda$ corrections in the SM. We need to specify the minimal FFs that overlap with two Higgses

$$
\begin{aligned}
F_{F F}\left(1_{i} 2_{j}^{*} 3_{a}^{-} 4_{b}^{-}\right) & =-2 \delta_{i}^{j} \delta_{A B}\langle 34\rangle^{2}, \\
F_{6}\left(1_{a} 2_{b} 3_{c} 4_{d}^{*} 5_{e}^{*} 6_{f}^{*}\right) & =6 \delta_{a}^{d} \delta_{b}^{e} \delta_{c}^{f}+\mathcal{P}_{d, e, f}, \\
F_{y}\left(1_{i} 2_{j}^{*} 3_{k} 4_{l}^{-} 5^{-}\right) & =\left(\delta_{i}^{j} \delta_{k}^{l}+\delta_{k}^{j} \delta_{i}^{l}\right)\langle 45\rangle, \\
F_{Q H}\left(1_{i} 2_{j}^{*} 3_{k}^{-} 4_{l}^{+}\right) & =-T_{j i}^{A} T_{k l}^{A}(\langle 31\rangle[14]-\langle 32\rangle[24]),
\end{aligned}
$$

where $\mathcal{P}_{X}$ means all the permutations of the previous term over the elements in $X$. The lower case and capital case indices in (2.17)-(2.20) are fundamental and adjoint indices of $\mathrm{SU}(2)$, respectively. For operators containing fermions or vectors, one can generate FF with different helicity configurations. Throughout the text we will chose to do the calculations with minimal FF that have mostly negative helicity particles. Due to CPT, if the calculation is done with the mostly positive helicity FF the results are the same.

In table 1 we provide the normalisation of the operators involved in these FFs. There we also provide the rest of minimal FFs that we will use throughout out the paper. Note that when using on-shell methods, a natural base of dimensions-six operators to use is the so called Warsaw basis [17], because in such basis all operators that vanish on-shell have been traded by higher point contact interactions [18-22].

Next we contract each FF in (2.17)-(2.20) with (2.7) and perform the basic tensor multiplications and $d \Omega_{2}$ integrals as in (2.6). Note that the calculation of the anomalous dimension of the FFs $F_{F F}, F_{6}$ and $F_{y}$ is rather simple because the integration over the

\footnotetext{
${ }^{5}$ The renormalization mixing pattern in $(2.15),(2.16)$ for $N=2$ can be understood using custodial symmetry and spurion analysis [15]. While $O_{\perp}$ transforms non-trivially, the quartic interactions and $O_{\|}$ are singlets under custodial transformation.
} 


\begin{tabular}{|lllll|}
\hline \multicolumn{3}{|c|}{ Operator } & MFF & \\
\hline$O_{3 F}$ & $\frac{f^{A B C}}{2 \cdot 3 !} F_{A \nu}^{\mu} F_{B \rho}^{\nu} \bar{F}_{C \mu}^{\rho}$ & $F_{3}\left(1_{A}^{-} 2_{B}^{-} 3_{C}^{-}\right)$ & $\langle 12\rangle\langle 23\rangle\langle 31\rangle$ & $i f^{A B C} / \sqrt{2}$ \\
$O_{F F}$ & $\frac{1}{2} H^{\dagger} H F_{\mu \nu}^{A} \bar{F}^{A \mu \nu}$ & $F_{F F}\left(1_{i} 2_{j}^{*} 3_{A}^{-} 4_{B}^{-}\right)$ & $\langle 34\rangle\langle 34\rangle$ & $-2 \delta_{i}^{j} \delta_{A B}$ \\
$O_{q F}$ & $\bar{Q} \sigma^{\mu \nu} T^{A} q H F_{\mu \nu}^{A}$ & $F_{q F}\left(1_{i}^{-} 2^{-} 3_{k} 4_{A}^{-}\right)$ & $\langle 14\rangle\langle 42\rangle$ & $2 \sqrt{2} T_{i k}^{A}$ \\
$O_{4 F_{1}}$ & $\left(\bar{Q}_{i} u\right) \epsilon_{i j}\left(\bar{Q}_{j} d\right)$ & $F_{4 F_{1}}\left(1_{i}^{-} 2^{-} 3_{j}^{-} 4^{-}\right)$ & $\langle 12\rangle\langle 34\rangle$ & $\epsilon_{i j}$ \\
$O_{y}$ & $|H|^{2} \bar{Q} q H$ & $F_{y}\left(1_{i} 2_{j}^{*} 3_{k} 4_{l}^{-} 5^{-}\right)$ & $\langle 45\rangle$ & $\delta_{i}^{j} \delta_{k}^{l}+\delta_{k}^{j} \delta_{i}^{l}$ \\
$O_{6}$ & $|H|^{6}$ & $F_{6}\left(1_{a} 2_{b} 3_{c} 4_{d}^{*} 5_{e}^{*} 6_{f}^{*}\right)$ & 1 & $6 \delta_{a}^{d} \delta_{b}^{e} \delta_{c}^{f}+\mathcal{P}_{d, e, f}$ \\
$O_{4 F_{2}}$ & $\left(\bar{Q} T^{A} \gamma^{\mu} Q\right)\left(\bar{Q} T^{A} \gamma_{\mu} Q\right)$ & $F_{4 F_{2}}\left(1_{i}^{-} 2_{j}^{-} 3_{k}^{+} 4_{l}^{+}\right)$ & $\langle 12\rangle[34]$ & $2 T_{i l}^{A} T_{j k}^{A}-2 T_{j l}^{A} T_{i k}^{A}$ \\
$O_{Q H}$ & $\left(\bar{Q} T^{A} \gamma^{\mu} Q\right)\left(i H^{\dagger} T^{A} \stackrel{\leftrightarrow}{D} \mu H\right)$ & $F_{Q H}\left(1_{i} 2_{j}^{*} 3_{k}^{-} 4_{l}^{+}\right)$ & $\langle 31\rangle[14]$ & $-2 T_{j i}^{A} T_{k l}^{A}$ \\
$O_{\perp}$ & $\left(H^{\dagger} D_{\mu} H\right)\left(D^{\mu} H\right)^{\dagger} H$ & $F_{\perp}\left(1_{i} 2_{j} 3_{k}^{*} 4_{l}^{*}\right)$ & $\langle 13\rangle[13]$ & $\delta_{i}^{l} \delta_{j}^{k}+\mathcal{P}_{1_{i}, 2_{j}}$ \\
$O_{\|}$ & $|H|^{2}\left(D^{\mu} H\right)^{\dagger}\left(D_{\mu} H\right)$ & $F_{\|}\left(1_{i} 2_{j} 3_{k}^{*} 4_{l}^{*}\right)$ & $\langle 14\rangle[14]$ & $\delta_{i}^{k} \delta_{j}^{l}+\mathcal{P}_{1_{i}, 2_{j}}$ \\
\hline
\end{tabular}

Table 1. The Standard Model dimension-six operators and its minimal form factors (MFF) — with the least number of particles. The MFFs are given by the product of the two rightmost columns. In the right most column we give the $\mathrm{SU}(2)_{L}$ and $\mathrm{SU}(3)_{c}$ tensors that compound the MFF - omitting the identity in colour space and flavour mixing matrices. $\bar{F}_{\alpha \beta}=F_{\alpha \beta}-i \epsilon_{\alpha \beta \mu \nu} F^{\mu \nu} / 2$, and $T^{A}$ is either of the generators of the SM gauge groups. We are not displaying operators whose MFF involves a straightforward modification of the tensor structure - in the rightmost column - of the operator shown. e.g. like $H^{\dagger} \sigma^{i} H B_{\mu \nu} W^{i \mu \nu}, i \tilde{H}^{\dagger} D_{\mu} H\left(u \gamma^{\mu} d\right)$ and $|H|^{2} \bar{Q} u \tilde{H}$, where $\tilde{H}=i \sigma_{2} H^{*}$.

rotation angles is trivial and all what is left is to perform the contraction of the $\mathrm{SU}(2)_{L}$ tensors. Indeed, for $F_{F F}$ we get

$$
-\frac{2 \lambda}{16 \pi^{2}}\left(\delta_{\alpha}^{j} \delta_{i}^{\beta}+\delta_{\alpha}^{\beta} \delta_{i}^{j}\right) \delta_{\beta}^{\alpha} \delta_{a b} 2\langle 12\rangle^{2}=\frac{2 \lambda}{16 \pi^{2}}(N+1) F_{F F}\left(1_{i} 2_{j}^{*} 3_{a}^{-} 4_{b}^{-}\right)
$$

The factor of 2 arises because there are two particle-antiparticle channels. Regarding the $\mathrm{O}_{6}$ self-renormalisation, there are two types of channels the particle-particle channel (or equivalently antiparticle-antiparticle) and the particle-antiparticle channel. There are 6 particle-particle type channels, each of them gives $-M\left(1_{a} 2_{b} ; 1_{a}^{\prime} 2_{b}^{\prime}\right) F_{6}\left(1_{a}^{\prime} 2_{b^{\prime}} 3_{c} 4_{d}^{*} 5_{e}^{*} 6_{f}^{*}\right)$ $=4 \lambda F_{6}\left(1_{a} 2_{b} 3_{c} 4_{d}^{*} 5_{e}^{*} 6_{f}^{*}\right)$. The second type, the particle-antiparticle channel, gives $-M\left(1_{a} 4_{d}^{*} ; 1_{a}^{\prime} 4_{d}^{* *}\right) F_{6}\left(1_{a}^{\prime} 2_{b} 3_{c} 4_{d}^{*} 5_{e}^{*} 6_{f}^{*}\right)+\mathcal{P}_{d, e, f}=14 \lambda F_{6}\left(1_{a} 2_{b} 3_{c} 4_{d}^{*} 5_{e}^{*} 6_{f}^{*}\right)$ for a Higgs doublet. There are 6 types of contributions like this. Thus, all in all we get $6(4+14) \lambda /\left(16 \pi^{2}\right)$, at leading order in $\lambda$. Regarding $F_{y}$, there are also two channels. The particle-particle gives $-M\left(1_{i} 3_{k} ; 1_{a}^{\prime} 3_{b}^{\prime}\right) F_{y}\left(1_{a}^{\prime} 2_{j}^{*} 3_{b}^{\prime} 4_{l}^{-} 5^{-}\right)=4 \lambda F_{y}\left(1_{i} 2_{j}^{*} 3_{k} 4_{l}^{-} 5^{-}\right)$. The second type, the particleantiparticle channel, gives $-M\left(1_{i} 2_{j}^{*} ; 1_{a}^{\prime} 2_{b}^{\prime *}\right) F_{y}\left(1_{a}^{\prime} 2_{b}^{\prime *} 3_{b} 4_{l}^{-} 5^{-}\right)+\mathcal{P}_{i k}=$ $2(N+3) \lambda F_{y}\left(1_{i} 2_{j}^{*} 3_{k} 4_{l}^{-} 5^{-}\right)$; there are two such terms because $\left|1_{a}^{\prime} 2_{b}^{*}\right\rangle$ and $\left|1_{b}^{\prime} 2_{a}^{\prime *}\right\rangle$ are distinct states. Adding all the channels we get $24 \lambda /\left(16 \pi^{2}\right)$ for the anomalous dimension of $F_{y}$. 
As for the calculation of $F_{Q H}$, there is only a particle-antiparticle channel

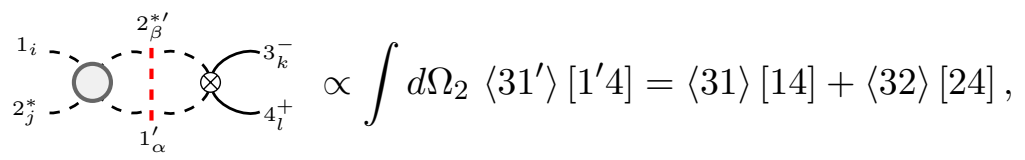

where the solid lines represent fermions. The former polynomial vanishes by momentum conservation $\sum_{i=1}^{4}|i\rangle\left[i \mid=0 .{ }^{6}\right.$ This is of course unsurprising since the mixing is only sensitive to the current $J_{\mu}^{A}=i H^{\dagger} T^{A} \partial_{\mu} H-i \partial_{\mu} H^{\dagger} T^{A} H$ in the operator $O_{Q H}=J_{\mu}^{A}\left(\bar{Q} \gamma^{\mu} T^{A} Q\right)$. The current $J_{\mu}^{A}$ is conserved (up to terms proportional to the $\mathrm{SU}(2)_{L}$ coupling $g$ ), hence it obeys a Ward identity and thus does not acquire an anomalous dimension proportional to $\lambda[1+O(g)]$.

All in all, we recover the order $\lambda$ anomalous dimension matrix of the dimensions-six operators

$$
\left(\begin{array}{l}
\gamma_{F F} \\
\gamma_{\perp} \\
\gamma_{\|} \\
\gamma_{6} \\
\gamma_{y} \\
\gamma_{\psi H}
\end{array}\right)=\frac{4 \lambda}{16 \pi^{2}}\left(\begin{array}{cccccc}
3 & & & & & \\
& 3 & & & & \\
& & 6 & & & \\
& & 27 & & \\
& & & 6 & \\
& & & & 0
\end{array}\right)\left(\begin{array}{l}
c_{F F} \\
c_{\perp} \\
c_{\|} \\
c_{6} \\
c_{y} \\
c_{\psi H}
\end{array}\right)
$$

where we only show the non-vanishing matrix elements. Eq. (2.23) is in agreement with [14-16].

\subsection{Contributions from gauge interactions}

Now we consider contributions to anomalous dimensions that are proportional to a gauge coupling squared. These contributions arise when an $O\left(g^{2}\right) \mathcal{M}$-matrix element is used in (2.6). In this case however, $\mathcal{M}$-matrix elements can have collinear and soft divergences, which generate a non-vanishing contribution to $\gamma_{\mathrm{IR}}$. We will therefore describe how the formalism must be used when IR divergences are present, first calculating the anomalous dimension of a conserved Noether current as a simple example. Then, we will calculate gauge contributions to the anomalous dimensions of $O_{\|}$and $O_{\perp}$.

\subsubsection{IR divergences}

In a gauge theory, the IR anomalous dimension has the following expression at the one-loop level:

$$
\gamma_{\mathrm{IR}}\left(s_{i j} ; \mu\right)=\sum_{i<j} T_{i}^{A} \cdot T_{j}^{A} \gamma_{\mathrm{cusp}} \log \frac{\mu^{2}}{-s_{i j}}+\sum_{i} \gamma_{i}^{\text {coll }},
$$

see e.g. refs. $[23,24]$. In $(2.24)$ the sum runs over ordered pairs of external legs, $T_{i}^{A}$ is a generator of the gauge group, and $\mu$ is the t'Hooft scale of dimensional regularisation. This form for $\gamma_{\mathrm{IR}}$ has also been confirmed to hold in a number of examples at two and three

\footnotetext{
${ }^{6}$ The corresponding diagram in an Effective Action calculation leads to $\partial|H|^{2} \bar{Q} \gamma Q \stackrel{\text { e.o.m. }}{\longrightarrow} y O_{y}+y O_{y}^{*}$, where $y$ is the Yukawa coupling and we have used the equations of motion. In the on-shell calculation, such order $\lambda y$ contribution comes from using $\left\langle H H^{\dagger} H \bar{Q} Q\right|$ as an outgoing state in (2.6).
} 
loops in QCD and $\mathcal{N}=4 \mathrm{SYM}$ (see [24] and references therein), and has been conjectured to hold at all subsequent loop orders. For our current purposes it suffices that (2.24) holds at one loop for either of the SM gauge groups with collinear dimensions given by

$$
\gamma_{g}^{\text {coll }}=-b_{0} \frac{g^{2}}{16 \pi^{2}}, \quad \gamma_{q}^{\text {coll }}=-3 C_{2} \frac{g^{2}}{16 \pi^{2}}, \quad \gamma_{H}^{\text {coll }}=-4 C_{2} \frac{g^{2}}{16 \pi^{2}},
$$

where $b_{0}$ is the one-loop beta function coefficient, $C_{2} \mathbb{1}=\sum_{A} T^{A} T^{A}$, and at one loop $\gamma_{\text {cusp }}=g^{2} /\left(4 \pi^{2}\right)$. The collinear dimensions $\gamma_{g / q}^{\text {coll }}$ are shown in [23] and $\gamma_{H}^{\text {coll }}$ can be extracted from [25]. Using (2.24) in (2.4) it can be shown that [3]

$$
\begin{aligned}
\gamma_{j i} F_{O_{j}}= & -\frac{1}{16 \pi^{2}} \int d \Omega_{2}\left(M\left(12 ; 1^{\prime} 2^{\prime}\right) F_{O_{i}}\left(1^{\prime} 2^{\prime} 3 \ldots n\right)+\frac{2 g^{2} T_{1}^{A} T_{2}^{A} F_{O_{i}}(123 \ldots n)}{\sin ^{2} \theta \cos ^{2} \theta}\right) \\
& +F_{O_{i}} \sum_{k} \gamma_{k}^{\text {coll }},
\end{aligned}
$$

where as in (2.4) we leave implicit the sum over permutations of ordered pairs of the $n$ external particles.

The FF of a Noether current that corresponds to an ungauged global symmetry has a vanishing UV anomalous dimension. Next, we will calculate the anomalous dimension of such a FF and confirm that it is zero to provide a good consistency check on (2.26). We have an ungauged global symmetry in the SM Higgs sector, associated with SU(2) rotations of the Higgs doublet once the $\mathrm{SU}(2)_{L}$ gauge coupling has been set to zero. The corresponding Noether current is $J_{\mu}^{A}=i\left(H^{\dagger} T^{A} \partial_{\mu} H-\partial_{\mu} H^{\dagger} T^{A} H\right)$, and its associated MFF is

$$
F_{J^{A}}\left(1_{i} 2_{j}^{*}\right)=-T_{i j}^{A}\left(\lambda_{1}^{\alpha} \tilde{\lambda}_{1}^{\dot{\alpha}}-\lambda_{2}^{\alpha} \tilde{\lambda}_{2}^{\dot{\alpha}}\right),
$$

where $T^{A}=\sigma^{A} / 2$. We will consider the contribution to the UV anomalous dimension of this FF coming from the SM Hypercharge interaction. To compute this contribution to $O\left(g^{\prime 2}\right)$, we need the tree-level $2 \rightarrow 2$ scattering matrix element for Higgs scalars due to the Hypercharge gauge interaction:

$$
M\left(1_{i} 2_{j} 3_{k}^{*} 4_{l}^{*}\right)=g^{\prime 2} Y_{H}^{2} \delta_{i}^{k} \delta_{j}^{l}\left(1-2 \frac{\langle 12\rangle\langle 34\rangle}{\langle 13\rangle\langle 24\rangle}\right)+g^{\prime 2} Y_{H}^{2} \delta_{i}^{l} \delta_{j}^{k}\left(1-2 \frac{\langle 12\rangle\langle 34\rangle}{\langle 23\rangle\langle 41\rangle}\right) .
$$

Evaluating (2.26) for the non-abelian current in (2.27), we have

$$
\begin{aligned}
\gamma_{J J} F_{J^{A}}\left(1_{i} 2_{j}^{*}\right)=-\frac{1}{16 \pi^{2}} \int d \Omega_{2}( & M\left(1_{i} 2_{j}^{*} ; 1_{\alpha}^{\prime} 2_{\beta}^{*^{\prime}}\right) F_{J^{A}}\left(1_{\alpha}^{\prime} 2_{\beta}^{*^{\prime}}\right)+M\left(1_{i} 2_{j}^{*} ; 1_{\alpha}^{*^{\prime}} 2_{\beta}^{\prime}\right) F_{J^{A}}\left(1_{\alpha}^{*^{\prime}} 2_{\beta}^{\prime}\right) \\
& \left.-\frac{g^{\prime 2} F_{J^{A}}\left(1_{i} 2_{j}^{*}\right)}{2 \sin ^{2} \theta \cos ^{2} \theta}\right)+F_{J^{A}}\left(1_{i} 2_{j}^{*}\right) \sum_{k} \gamma_{k}^{\text {coll }}
\end{aligned}
$$

where we have used $T_{1}^{A} T_{2}^{A}=-1 / 4$ for the hypercharge generators $Y_{H}=1 / 2$ acting on a Higgs scalar and its antiparticle. The integral in (2.29) is evaluated as usual. After performing the angular rotations and basic integrations, we find that it equals $2 g^{\prime 2} F_{J^{A}}\left(1_{i} 2_{j}^{*}\right) / 16 \pi^{2}$. Next, using the result for the collinear dimension of scalars from (2.25), and that $C_{2}=1 / 4$, 
we find that the final term in eq. (2.29) equals $-2 g^{\prime 2} F_{J^{A}}\left(1_{i} 2_{j}^{*}\right) / 16 \pi^{2}$. We have therefore demonstrated that the required result $\gamma_{J J}=0$, can be recovered using this formalism.

In passing, we note that if the same exercise is instead carried out for the Noether current associated with the gauged $\mathrm{U}(1)_{Y}$ symmetry, we get a non-vanishing anomalous dimension. This is because renormalisation of the naive Noether current occurs when the corresponding abelian symmetry is gauged. However, an improved current can be defined in this case that is both conserved and non-renormalised [26].

Having done some simple checks of (2.26), we now compute instances of gauge contributions to the SM dimension-six anomalous dimensions.

\subsubsection{Gauge mixing}

Coming back to the operators $O_{\|}$and $O_{\perp}$, let us discuss the mixing due to the $\mathrm{U}(1)_{Y^{-}}$ Hypercharge. We will use (2.26) to calculate the matrix of anomalous dimensions, which is needed as there is both operator mixing and a non-vanishing IR anomalous dimension for this interaction and choice of FFs. In this formula, we will plug in the matrix element for scalar scattering due to the Hypercharge interaction, given by (2.28).

Begin by first taking $F_{\perp}$ in the integral. Just like the corresponding $O(\lambda)$ calculation in section 2.1, there will be three independent channels that contribute to the integral, corresponding to different pairs of scalars crossing the cut. The integral for the first channel gives

$$
\begin{aligned}
- & \frac{1}{16 \pi^{2}} \int d \Omega_{2}\left(M\left(1_{i} 2_{j} ; 1_{\alpha}^{\prime} 2_{\beta}^{\prime}\right) F_{\perp}\left(1_{\alpha}^{\prime} 2_{\beta}^{\prime} 3_{k}^{*} 4_{l}^{*}\right)+\frac{g^{\prime 2} F_{\perp}\left(1_{i} 2_{j} 3_{k}^{*} 4_{l}^{*}\right)}{2 \sin ^{2} \theta \cos ^{2} \theta}\right) \\
& =-\frac{g^{\prime 2}}{64 \pi^{2}} \delta_{i}^{l} \delta_{j}^{k}\{5\langle 13\rangle[31]-3\langle 14\rangle[41]\}-\frac{g^{\prime 2}}{64 \pi^{2}} \delta_{j}^{l} \delta_{i}^{k}\{5\langle 14\rangle[41]-3\langle 13\rangle[31]\},
\end{aligned}
$$

where the Higgs Hypercharge value $Y_{H}=1 / 2$ has been inputted. Individually, the two terms appearing in the integral in eq. (2.30) are divergent, but their sum is finite. The integral for the second channel is

$$
\begin{aligned}
& -\frac{1}{16 \pi^{2}} \int d \Omega_{2}\left(M\left(1_{i} 3_{k}^{*} ; 1_{\alpha}^{\prime} 3_{\beta}^{* \prime}\right) F_{\perp}\left(1_{\alpha}^{\prime} 2_{j} 3_{\beta}^{* \prime} 4_{l}^{*}\right)+M\left(1_{i} 3_{k}^{*} ; 1_{\alpha}^{* \prime} 3_{\beta}^{\prime}\right) F_{\perp}\left(1_{\alpha}^{* \prime} 2_{j} 3_{\beta}^{\prime} 4_{l}^{*}\right)\right. \\
& \left.-\frac{g^{\prime 2} F_{\perp}\left(1_{i} 2_{j} 3_{k}^{*} 4_{l}^{*}\right)}{2 \sin ^{2} \theta \cos ^{2} \theta}\right) \\
& =\frac{2 g^{\prime 2}}{64 \pi^{2}} \delta_{i}^{l} \delta_{j}^{k}\langle 13\rangle[31]+\frac{g^{\prime 2}}{64 \pi^{2}} \delta_{j}^{l} \delta_{i}^{k}\left\{\left(\frac{N}{3}+3\right)\langle 13\rangle[31]+\left(\frac{2 N}{3}+8\right)\langle 14\rangle[41]\right\} .
\end{aligned}
$$

The integral for the third channel can be found from the second by making the substitutions $3 \leftrightarrow 4$ and $k \leftrightarrow l$. Finally, multiplying each of these three channels by two (to include the crossing symmetric contributions) and adding them gives the total value for the integral in (2.26). Using the equations for the FFs in (2.8) and (2.9), we can express this total as

$$
\frac{g^{\prime 2}}{16 \pi^{2}}\left(\frac{N}{3}+\frac{5}{2}\right) F_{\perp}\left(1_{i} 2_{j} 3_{k}^{*} 4_{l}^{*}\right)+\frac{g^{\prime 2}}{16 \pi^{2}}\left(\frac{N}{6}+3\right) F_{\|}\left(1_{i} 2_{j} 3_{k}^{*} 4_{l}^{*}\right)
$$

Using (2.25), we find that the collinear contribution is $\sum \gamma^{\text {coll }}=-4 g^{\prime 2} / 16 \pi^{2}$. Then by matching coefficients of $F_{\perp}$ and $F_{\|}$in equation (2.26), we can extract order $g^{\prime}$ values for 
two elements of the anomalous dimension matrix $-\gamma_{\perp}=g^{\prime 2}(N / 3-3 / 2) c_{\perp} / 16 \pi^{2}$ and $\gamma_{\|}=g^{\prime 2}(N / 6+3) c_{\perp} / 16 \pi^{2}$.

By repeating this procedure except inputting $F_{\|}$to the integral in (2.26), the remaining two elements of the anomalous dimension matrix can be found. After combining the three channels in the same way as before, the integral term is

$$
\frac{g^{\prime 2}}{16 \pi^{2}}\left(\frac{10}{3} F_{\perp}\left(1_{i} 2_{j} 3_{k}^{*} 4_{l}^{*}\right)+\frac{8}{3} F_{\|}\left(1_{i} 2_{j} 3_{k}^{*} 4_{l}^{*}\right)\right),
$$

independently of $N$. After adding the collinear contribution and matching coefficients of $F_{\perp}$ and $F_{\|}$in eq. (2.26), we can find the remaining two elements of the $O_{\|}$and $O_{\perp}$ anomalous dimension matrix at order $g^{\prime 2}$. After setting $N=2$ for the Higgs doublet, the final result is

$$
\left(\begin{array}{c}
\gamma_{\perp} \\
\gamma_{\|}
\end{array}\right)=\frac{g^{\prime 2}}{16 \pi^{2}}\left(\begin{array}{cc}
-\frac{5}{6} & \frac{10}{3} \\
\frac{10}{3} & -\frac{4}{3}
\end{array}\right)\left(\begin{array}{l}
c_{\perp} \\
c_{\|}
\end{array}\right),
$$

in agreement with previous literature $[15,27] .{ }^{7}$ By similar means one can compute the mixing due to the $\mathrm{SU}(2)_{L}$ gauge interactions. However, such order $g^{2}$ contributions are diagonal because, due to custodial symmetry, the RG mixing between $O_{\|}$and $O_{\perp}$ must be proportional to the Hypercharge coupling $g^{\prime 2}[15]$.

\section{$2.3 \quad$ Non-minimal form factors}

In the previous sections we have only encountered minimal form factors, i.e. FF that do not vanish at zero order in the SM couplings. This is because we focused on transitions between operators that have the same number of external fields. Instead, if the transition between operators increases the number of particles, we will potentially have contributions from a $2 \rightarrow 2$ S-matrix element acting on a non-minimal form factor.

Next we compute an illustrative example consisting on the dipole renormalisation by the $F^{3}$ operator. For concreteness we focus on the $F^{3}$ and dipole involving the gluon field strength. The MFFs that we need are ${ }^{8}$

$$
F_{3 G}\left(1_{A}^{-} 2_{B}^{-} 3_{C}^{-}\right)=\frac{i}{\sqrt{2}} f^{A B C}\langle 12\rangle\langle 23\rangle\langle 31\rangle, \quad F_{q G}\left(1_{i}^{-} 2_{j}^{-} 34_{A}^{-}\right)=2 \sqrt{2} T_{i j}^{A}\langle 14\rangle\langle 42\rangle,
$$

where in $F_{q G}$ we only show the color index, because the SU(2) indices are diagonal throughout the calculation. There are three different contributions,

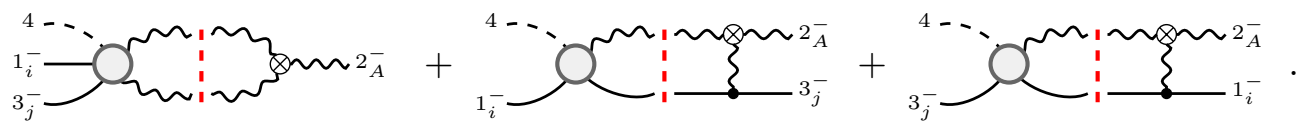

The first diagram in (2.36) involves a 5-point scattering amplitude. The amplitude between two fermions, a scalar and two gluons with all-plus helicity vanishes:

\footnotetext{
${ }^{7}$ The calculation of [15] was done in a basis where $O_{B} \equiv\left(H^{\dagger} D_{\nu} H\right) \partial_{\mu} B^{\mu \nu}$ is kept as an independent operator. After rotating away such operator and rotating the operators $\left\{O_{H}, O_{T}\right\}$ (there defined) into $\left\{O_{\|}, O_{\perp}\right\}$ full agreement is found. An early calculation of the RG mixing from $O_{H}$ to $O_{T}$ can be found in [28].

${ }^{8}$ Where we used the convention $\epsilon_{a \dot{a}}^{-}=-\sqrt{2} \lambda_{a} \tilde{\mu}_{\dot{a}} /[\tilde{\lambda}, \tilde{\mu}]$.
} 
$M\left(\psi^{+} \psi^{+} \phi g^{+} g^{+}\right)=0$. Therefore the only contribution is from the amplitude with negative helicity fermions $M\left(\psi^{-} \psi^{-} \phi g^{+} g^{+}\right)$, which is a potential contribution to the dipole $F_{q G}\left(1_{i}^{-} 2_{j}^{-} 34_{A}^{-}\right)$in $(2.35)$. The 5 -point amplitude is

$$
M\left(1_{i}^{-} 2_{A}^{+} 3_{B}^{+} 4_{j}^{-} 5\right)=-2 y g_{s}^{2}\left(T^{A} T^{B}\right)_{i j} \frac{\langle 14\rangle^{2}}{\langle 12\rangle\langle 23\rangle\langle 34\rangle}-2 y g_{s}^{2}\left(T^{B} T^{A}\right)_{i j} \frac{\langle 14\rangle^{2}}{\langle 13\rangle\langle 32\rangle\langle 24\rangle} .
$$

We need to perform the convolution of this amplitude with $F_{3 G}$ in (2.35),

$$
-\frac{1}{16 \pi^{2}} \int \mathrm{d} \Omega_{2} M\left(1_{i}^{-} 3_{j}^{-} 4 ; x_{\mathrm{X}}^{-} y_{\mathrm{Y}}^{-}\right) F_{3 G}\left(x_{\mathrm{X}}^{-} y_{\mathrm{Y}}^{-} 2_{A}^{-}\right)=-\frac{y g_{s}^{2}}{16 \pi^{2}} \frac{N_{c} T_{i j}^{A}}{\sqrt{2}} 2\langle 13\rangle^{2} \int \mathrm{d} \Omega_{2} \frac{\langle y 2\rangle\langle 2 x\rangle}{\langle 1 x\rangle\langle y 3\rangle}
$$

where we used $-i f^{A B C} T^{A} T^{B}=\frac{1}{2} N_{c} T^{C}$, and a factor of two arises because both color orderings in (2.37) give the same integral since it is invariant under $x \leftrightarrow y$. Now we need to rotate the internal spinors in terms of the three external ones. We rotate $|x\rangle$ and $|y\rangle$ in terms of spinors $|a\rangle$ and $|b\rangle$ such that they are on-shell and $p_{a}+p_{b}=p_{1}+p_{3}+p_{4}$, a simple choice is [3]

$$
|a\rangle=|1\rangle \sqrt{\frac{s_{134}}{s_{13}+s_{14}}}, \quad|b\rangle=(|3\rangle[13]+|4\rangle[14]) \frac{1}{\sqrt{s_{13}+s_{14}}}
$$

Next we rotate $(|x\rangle,|y\rangle)=R(\theta, \phi) .(|a\rangle,|b\rangle)$ like in $(2.5)$ and we are lead to

$$
\int_{0}^{\pi / 2} 2 c_{\theta} s_{\theta} \mathrm{d} \theta \int \frac{\mathrm{d} \phi}{2 \pi} \frac{\left(\langle b 2\rangle c_{\theta}+s_{\theta} e^{-i \phi}\langle a 2\rangle\right)\left(\langle 2 a\rangle c_{\theta}-s_{\theta} e^{i \phi}\langle 2 b\rangle\right)}{-s_{\theta} e^{i \phi}\langle 1 b\rangle\left(\langle b 3\rangle c_{\theta}+s_{\theta} e^{-i \phi}\langle a 3\rangle\right)} .
$$

A convenient way to perform the $\phi$ and $\theta$ integrals is to change variables to $z \equiv e^{i \phi}$ and $t \equiv \tan \theta$. In this way, the denominator of the integrand is a product of terms like $(z-\langle i j\rangle /\langle i k\rangle t)$ so the $z$ integral picks the residues inside the unit circle and the integral on $t$ scans the position of those residues. The integral in (2.40) is

$$
-2 \frac{\langle 2 b\rangle\langle 2 a\rangle}{\langle 1 b\rangle\langle 3 b\rangle} \int_{0}^{\infty} \frac{\mathrm{d} t}{\left(1+t^{2}\right)^{2}} \oint_{|z|=1} \frac{\mathrm{d} z}{2 \pi i} \frac{\left(z+t r_{2}\right)\left(1-z t / r_{2}\right)}{z^{2}\left(z+t r_{3}\right)}
$$

where we have defined $r_{i}=\langle i a\rangle /\langle i b\rangle$. After performing the integrals, we use the expressions in (2.39), and after a lengthy manipulation of the result using Schouten identities $[\xi i][j k]+$ $[\xi j][k i]+[\xi k][i j]=0$ we get

$$
\begin{aligned}
(2.41)= & \frac{\langle 12\rangle\langle 23\rangle}{\langle 13\rangle^{2}}\left(2-\log \frac{\left(s_{13}+s_{14}\right)\left(s_{13}+s_{34}\right)}{s_{14} s_{34}}\right) \\
& +\frac{\langle 12\rangle^{2}\langle 34\rangle}{\langle 14\rangle\langle 13\rangle^{2}} \frac{s_{14}}{s_{13}+s_{14}}+\frac{\langle 14\rangle\langle 23\rangle^{2}}{\langle 34\rangle\langle 13\rangle^{2}} \frac{s_{34}}{s_{13}+s_{34}} .
\end{aligned}
$$

Next we compute the right diagram in (2.36), which involves the following four-particle amplitude and non-minimal FF

$$
\begin{gathered}
M\left(1_{i}^{-} 2_{A}^{+} 3_{j}^{-} 4\right)=y g_{s} \sqrt{2} T_{i j}^{A} \frac{\langle 13\rangle^{2}}{\langle 12\rangle\langle 23\rangle} \\
F_{3 G}\left(1_{i}^{-} 2_{A}^{-} 3_{B}^{-} 4_{j}^{+}\right)=-i g_{s} f^{A B C} T_{i j}^{C} \frac{\langle 12\rangle\langle 23\rangle\langle 31\rangle}{\langle 34\rangle} .
\end{gathered}
$$


Convoluting this scattering matrix element with the $\mathrm{FF}$ we get

$$
\begin{aligned}
& -\frac{2}{16 \pi^{2}} \int \mathrm{d} \Omega_{2} M\left(1_{i}^{-} 4 ; x_{\mathrm{X}}^{-} y_{k}^{+}\right) F_{3 G}\left(2_{A}^{-} x_{\mathrm{X}}^{-} 3_{j}^{-} y_{k}^{+}\right) \\
& =-\frac{y g_{s}^{2}}{16 \pi^{2}} \frac{N_{c} T_{i j}^{A}}{\sqrt{2}} 2 \frac{\langle 23\rangle}{\langle 14\rangle} \int \mathrm{d} \Omega_{2} \frac{\langle 1 y\rangle^{2}\langle 2 x\rangle\langle 3 x\rangle}{\langle 1 x\rangle\langle 3 y\rangle} .
\end{aligned}
$$

The factor of two comes because there are two channels corresponding to propagating either $\left|x_{\mathbf{X}}^{-} y_{k}^{+}\right\rangle$or $\left|y_{k}^{+} x_{\mathbf{X}}^{-}\right\rangle$, that give the same value. Next we rotate $(|x\rangle,|y\rangle)=R(\theta, \phi) .(|1\rangle,|4\rangle)$ and perform again the change of variables into $z \equiv e^{i \phi}$ and $t \equiv \tan \theta$. We are left with the following integral

$$
\begin{aligned}
2 \int_{0}^{\infty} & \frac{\mathrm{d} t}{\left(1+t^{2}\right)^{3}} \oint_{|z|=1} \frac{\mathrm{d} z}{2 \pi i} \frac{\left(1+z t \frac{\langle 24\rangle}{\langle 12\rangle}\right)\left(\frac{\langle 13\rangle}{\langle 34\rangle}+t z\right)}{z\left(t \frac{\langle 13\rangle}{\langle 34\rangle}-z\right)} \\
& =\left(-\frac{3}{2}+\log \frac{s_{13}+s_{34}}{s_{34}}-\frac{\langle 14\rangle\langle 23\rangle}{\langle 12\rangle\langle 34\rangle} \frac{s_{34}}{s_{13}+s_{34}}\right)
\end{aligned}
$$

The final result for this contribution is $-\frac{y g_{s}^{2}}{16 \pi^{2}} \frac{N_{c} T_{i j}^{A}}{\sqrt{2}} 2\langle 12\rangle\langle 23\rangle \times(2.46)$.

The third contribution in (2.36) comes from exchanging 1 and 3 in the second contribution. Finally adding the three contributions, we see that the log's and rational functions cancel and we are left with the contact interaction

$$
\frac{y g_{s}^{2}}{16 \pi^{2}} \frac{N_{c}}{2} T_{i j}^{A} 2 \sqrt{2}\langle 12\rangle\langle 23\rangle,
$$

which we easily recognise as the dipole MFF in (2.35). Thus the anomalous dimension is

$$
\gamma_{q G \leftarrow 3 G}=\frac{g_{s}^{2} y}{16 \pi^{2}} \frac{N_{c}}{2}
$$

in agreement with [29].

In passing we note that if we do the calculation with the positive helicity gluons $F_{3 G}\left(1_{A}^{+} 2_{B}^{+} 3_{C}^{+}\right)$, i.e. the $\mathrm{CPT}$ related $\mathrm{FF}$, we get the complex conjugate dipole. The Wilson coefficient in $c_{3 G} O_{3 G}$ is taken as complex and thus we generate the operator $O_{q G}$ with a complex coefficient, namely the $\mathrm{CP}$ even and $\mathrm{CP}$ odd dipole.

\section{Structure of the anomalous dimension matrix}

The on-shell formalism that we have been using suggests classifying the operators — or minimal $\mathrm{FF}$ - in five classes,

$$
\langle\cdot\rangle^{3},\langle\cdot\rangle^{2}, \quad\langle\cdot\rangle, \quad 1 \text { and }\langle\cdot\rangle[\cdot]
$$

depending on the number of $\lambda$ and $\widetilde{\lambda}$ 's. For instance $F_{3 G}\left(1_{A}^{-} 2_{B}^{-} 3_{C}^{-}\right) \propto\langle 12\rangle\langle 23\rangle\langle 31\rangle \sim\langle\cdot\rangle^{3}$ and therefore is of the first class, see table 1 for the rest of FFs. The total number of spinors and dimensional analysis determines the number of particles involved in each FF. ${ }^{9}$

\footnotetext{
${ }^{9}$ This coincides with the classifications in [30, 31].
} 


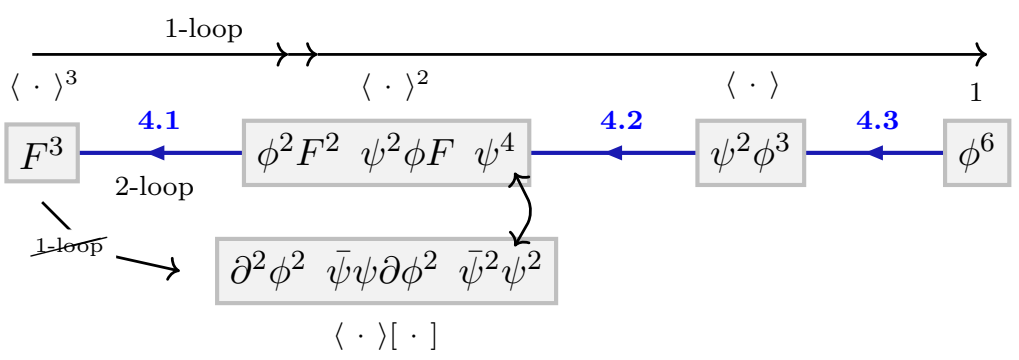

Figure 1. One-loop RG mixing can only occur from operators renormalising operators in boxes to the right or in the same box, with the only exception of the four-fermion of type $\langle\cdot\rangle^{2}$ and type $\langle\cdot\rangle[\cdot]$ that do mix. One-loop transitions from $\langle\cdot\rangle^{3}$-type operators to $\langle\cdot\rangle[\cdot]$ is forbidden by helicity selection rules. These patterns are summarised by the black arrows in the figure. With blue arrows we indicate the two-loop anomalous dimensions that are computed in the sections indicated on top.

The on-shell formalism makes very transparent the pattern of the anomalous dimensions matrix, namely the non-vanishing entries of the matrix. When mixing from $O_{i}$ to $O_{j}$, the phase-space integral will remove two legs from the form factor $F_{O_{i}}$ while the $S_{m \leftarrow 2}$ scattering matrix element generates $m \geqslant 2$ legs. Thus $O_{j}$ has, the same number of external fields or more, but never less than $O_{i}$. Therefore operators can only renormalise, at one-loop, operators with equal or more fields. This is an instance of the more general theorem in [32].

The RG mixing between the two four-particle classes $-\langle\cdot\rangle^{2}$ and $\langle\cdot\rangle[\cdot]$ - requires the 2 to 2 S-matrix element to have a total net helicity $\sum_{i} h_{i} \neq 0$ with all particles outgoing. In the SM there is a unique four-point tree-level amplitude that has net helicity, namely the four-fermion amplitude proportional to the up and down Yukawas $y_{u} y_{d}[30,33]$. Therefore the only allowed mixing between operators from class $\langle\cdot\rangle^{2}$ and $\langle\cdot\rangle[\cdot]$ is between the four-fermion operators $\psi^{4}$ and $\psi^{2} \bar{\psi}^{2}$. This effect was previously explained using SUSY as a spurious symmetry, since the up and down Yukawa interactions are not simultaneously holomorphic [34, 35]. Finally, helicity selection rules [30], or accidental supersymmetry [35], also exclude one-loop mixing from operators in $\langle\cdot\rangle^{3}$, into $\langle\cdot\rangle[\cdot] \cdot{ }^{10}$

While chunks of the SM dimensions-six operators' anomalous dimensions have been computed in many papers in the literature, see e.g. [14, 37-42] and [15, 43] for the anomalous dimensions relevant for Higgs and Electroweak physics, the full matrix has been computed in $[16,27,44,45]$. With this we conclude the summary of the one-loop pattern, see figure 1 for an outline.

A particularly interesting class of two-loop anomalous dimensions consists of those RG mixings that are forbidden at one-loop. We distinguish two types of such two-loop RG mixings. The first type are those two loop RG mixings that decrease the number of particles by a unit, namely an operator with $\ell$ legs can mix, at two loops, with an operator with $\ell-1$ legs. These transitions occur in the direction opposite to the long 1-loop arrow in figure 1. The second type are those two loop RG mixings that are forbidden at one loop

\footnotetext{
${ }^{10}$ Helicity selection rules also imply that the corresponding one-loop diagram vanishes up to a rational function of the kinematical variables; in many cases the rational factor vanishes as well [36], see also below.
} 
solely by selection rules (but not by leg counting). Those would be two loop transitions from $\langle\cdot\rangle^{3} \rightarrow\langle\cdot\rangle[\cdot]$ and two loop transitions among the four-particle operators of the classes $\langle\cdot\rangle[\cdot]$ and $\langle\cdot\rangle^{2}$.

In this work we will investigate the two-loop anomalous dimensions indicated by blue arrows in figure 1. These anomalous dimensions arise from the following type of diagrams

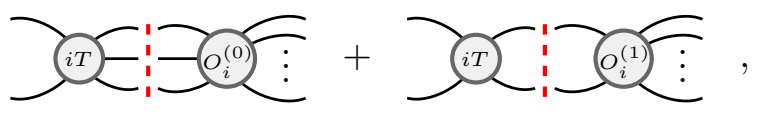

where the on-shell particle lines denote any allowed SM particle and the scattering element of each term is evaluated at tree-level. Indeed, under the three-particle cut of the first term of (3.2), three particles are removed from the operator $O_{i}$ while the $S_{2 \leftarrow 3}$ scattering matrix element produces two particles. By means of this process an operator with $\ell$ legs mixes, at two loops, with an operator with $\ell-1$ legs. There are no contributions that involve a 1-loop (or higher loop) scattering matrix element. Indeed, at two-loop order in the anomalous dimension matrix, such 1-loop scattering matrix element can only be contracted with two particles of the $\mathrm{FF}$ and thus does not decrease the number of particles in $O_{i}$. Finally, in principle we can also reduce the number of legs in the operator by closing the particles of the FF into a loop, this is indicated by the second term in (3.2). However, in the next section we will show that the second term in (3.2), involving the one-loop FF, vanishes in the two-loop mixing transitions that decrease the number of legs - the disappearance of this term was noted before in ref. [32].

In conclusion, for this class of anomalous dimensions, the only contribution comes from a tree-level S-matrix element, a tree-level FF and a three-particle cut (first term in (3.2)). They can be efficiently calculated and therefore the on-shell method is well suited for computing the leading RG mixings that shorten the length of the operator.

\section{The 'easy' two-loop anomalous dimensions}

In the following we compute the two-loop anomalous dimensions of the RG mixing shown by blue arrows in figure 1. This is a higher loop order calculation of the r.h.s. of (1.7) than what we have encountered so far. However it is still order "(0)" because, as we will see, such two-loop calculations lead to a Lorentz invariant polynomial in the spinors $\lambda, \tilde{\lambda}$. Thus, according to the l.h.s. of (1.7), its coefficient should be interpreted as the anomalous dimension $\gamma$ because $\mu \partial_{\mu}$ picks up the coefficient multiplying a single leading logarithm. ${ }^{11}$

The contributions coming from the first diagram in (3.2), consisting in $(-1 / \pi)$ times the three-particle phase space integral of a matrix element and a form factor, can be written as

$$
\frac{\langle 12\rangle[12]}{\left(16 \pi^{2}\right)^{2} 3 !} \int d \Omega_{3} M\left(12 ; 1^{\prime} 2^{\prime} 3^{\prime}\right) F_{O_{i}}\left(1^{\prime} 2^{\prime} 3^{\prime} 4 \ldots n\right),
$$

\footnotetext{
${ }^{11} \mathrm{Had}$ the one-loop not vanished, we would have needed to expand $e^{-i \pi D}$ to second order to get rid of the leading $\log ^{2}$ term and disentangled the coefficient of the one-loop and the two-loop single log.
} 
where the spinors in the integrand are written in terms the two external spinor by the following rotation

$$
\left(\begin{array}{l}
\lambda_{1}^{\prime} \\
\lambda_{2}^{\prime} \\
\lambda_{3}^{\prime}
\end{array}\right)=\left(\begin{array}{cc}
\cos \theta_{1} & -e^{i \phi} \cos \theta_{3} \sin \theta_{1} \\
\cos \theta_{2} \sin \theta_{1} & e^{i \phi}\left(\cos \theta_{1} \cos \theta_{2} \cos \theta_{3}-e^{i \delta} \sin \theta_{2} \sin \theta_{3}\right) \\
\sin \theta_{1} \sin \theta_{2} & e^{i \phi}\left(\cos \theta_{1} \cos \theta_{3} \sin \theta_{2}+e^{i \delta} \cos \theta_{2} \sin \theta_{3}\right)
\end{array}\right)\left(\begin{array}{c}
\lambda_{1} \\
\lambda_{2}
\end{array}\right),
$$

and the measure is $d \Omega_{3}=4 \cos \theta_{1} \sin ^{3} \theta_{1} d \theta_{1} 2 \cos \theta_{2} \sin \theta_{2} d \theta_{2} 2 \cos \theta_{3} \sin \theta_{3} d \theta_{3} \frac{d \delta}{2 \pi} \frac{d \phi}{2 \pi}[3,13]$. The $1 / 3$ ! in (4.1) arises only when considering the phase space for three identical particles. Further details on the phase-space integral can be found in appendix A.

\section{$4.1\langle\cdot\rangle^{2} \rightarrow\langle\cdot\rangle^{3}: 4$-point to 3-point}

We start by computing the transitions from four-field operators into three-field operators. There are only two structurally different contributions at two loops, namely the $\phi^{2} F^{2} \rightarrow F^{3}$ and the $\psi^{2} \phi F \rightarrow F^{3}$ mixings. We start with the former contribution. In the SM this transition is only possible when the gauge field belongs to the $\mathrm{SU}(2)_{L}$, thus we compute the RG mixing from the operator $O_{W W}$ to $O_{3 W}$, see table 1 with $F=W$. The corresponding MFFs are

$$
\begin{aligned}
F_{3 W}\left(1_{A}^{-} 2_{B}^{-} 3_{C}^{-}\right) & =\frac{i}{\sqrt{2}} f^{A B C}\langle 12\rangle\langle 23\rangle\langle 31\rangle \\
F_{W W}\left(1_{i} 2_{A}^{-} 3_{B}^{-} 4_{j}^{*}\right) & =-2 \delta_{i}^{j} \delta^{A B}\langle 23\rangle^{2} .
\end{aligned}
$$

We find the following potential contributions

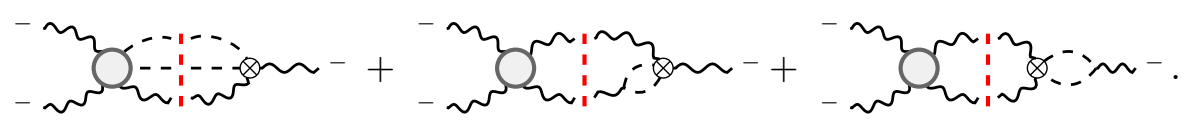

We have excluded one loop FFs that are contracted with the amplitude $H_{i} H_{j} \rightarrow W^{-} W^{-}$, because this process is zero at tree-level in the SM. Next, note that the one-loop FFs in (4.5) vanish. Indeed, the loop is given by $\int d^{d} \ell_{\frac{\left.1(\ell+p / 2)^{2}-i \epsilon\right]\left[(\ell-p / 2)^{2}-i \epsilon\right]}{}}=0 .{ }^{12}$ We are left with the first term in (4.5), involving a three-particle phase-space integration between a tree-level matrix element and a minimal FF. The five-point amplitude is given by

$$
M\left(1_{i} 2_{A_{2}}^{-} 3_{A_{3}}^{-} 4_{A_{4}}^{+} 5_{j}^{*}\right)=2 \sqrt{2} g^{3}[14]^{2}[45]^{2}\left(\frac{\left(T^{A_{2}} T^{A_{3}} T^{A_{4}}\right)_{i j}}{[12][23][34][45][51]}+\text { perms. }\{2,3,4\}\right),
$$

which has to be contracted with the MFF in (4.4),

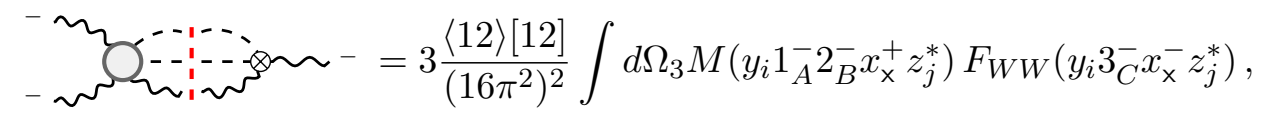

where we denoted by $x, y$ and $z$ the momenta of the spinors we are integrating over. In (4.7) we included a factor of 3 that accounts for all possible ways to contract the external state

\footnotetext{
${ }^{12}$ The same integral appeared recently in [32] when computing the two-loop mixing of $O_{6}$ into $O_{3 W}$.
} 
$\left\langle 1_{A}^{-} 2_{B}^{-} 3_{C}^{-}\right|$with the $M \otimes F$. The integral is done by rotating $\lambda_{x}, \lambda_{y}, \lambda_{z}$ in terms of $\lambda_{1}, \lambda_{2}$ as in (4.2). The integrand of (4.7) involves permutations of $[i j k] \equiv \frac{[x y]^{2}[x z]^{2}\langle x 3\rangle^{3}}{[y i][i j][j k][k z][z y]}$, with $i, j, k=1,2, x$, multiplied by the corresponding trace of $T^{A}$ 's matrices, generated from the contraction of the color structure in (4.6) with the $\delta_{i j}$ of the $F_{W W}$ form factor. The integral measure is invariant under $y \leftrightarrow z$, which is implemented with the change of variables $\left(\theta_{3}, \delta\right) \rightarrow\left(\pi / 2-\theta_{3}, \delta+\pi\right)$. Therefore, we can reverse the ordering of the arguments to get $[i j k]=-[k j i]$, and write the integrands proportional to $\operatorname{tr}\left(T^{A} T^{B} T^{C}\right)$ in terms of the ones proportional to $\operatorname{tr}\left(T^{C} T^{B} T^{A}\right)$. Thus we are lead to

$$
(4.7)=-\frac{\langle 12\rangle[12]}{\left(16 \pi^{2}\right)^{2}} 6 \sqrt{2} g^{3} i f^{A B C} \int d \Omega_{3}([12 x]+[x 12]+[2 x 1]),
$$

where we have used $\operatorname{tr}\left(T^{A}\left[T^{B}, T^{C}\right]\right)=i f^{A B C} / 2$ and a factor of 3 comes from the permutations in (4.7). The angular integrals in (4.8) are given by

$$
\int d \Omega_{3}[12 x]=\int d \Omega_{3}[x 12]=\frac{1}{2} \frac{\langle 13\rangle\langle 23\rangle}{[12]}, \quad \int d \Omega_{3}[2 x 1]=\frac{3}{2} \frac{\langle 13\rangle\langle 23\rangle}{[12]} .
$$

From this we read the two loop anomalous dimension

$$
\gamma_{3 W \leftarrow W W}=30 \frac{g^{3}}{\left(16 \pi^{2}\right)^{2}} .
$$

Next we compute a transition of the type $\psi^{2} \phi F \rightarrow F^{3}$. In particular we compute the renormalisation of the pure glue operator $O_{3 G}$ by the gluon dipole $O_{q G}$. This is the opposite mixing direction that we have computed in section 2.3. The MFFs are already given in (2.35). There are the following possible contributions

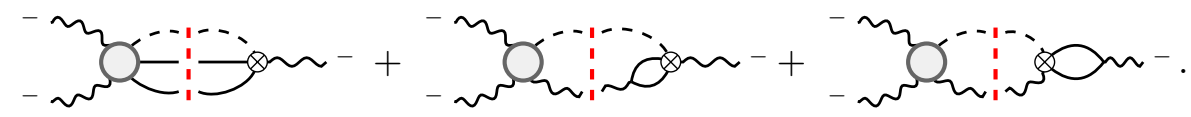

However, the one loop FF vanishes because, due to the presence of $\sigma_{\mu \nu}$ in the dipole vertex, the loop of fermions involves the trace of an odd number of $\gamma^{\mu}$-matrices. We have excluded one-loop FFs that are contracted with the amplitudes $\psi^{+} \psi^{+} \rightarrow g^{-} g^{-}$or $\phi \phi \rightarrow g^{-} g^{-}$ which do not exist, at tree-level, in the SM Lagrangian. Again, the contribution reduces to compute the first term in (4.11) which involves a tree-level matrix element and a minimal FF. The five-point scattering amplitude has already appeared in (2.37). Since in the current case the $\mathrm{SU}(2)$ indices are relevant, they are denoted by denoted by $i, j$, while the color ones by $\alpha, \beta$. Both the amplitude and FF proportional to the trivial factor $\delta_{i j}$. The convolution of the amplitude and the MFF is

$$
\int \mathrm{d} \Omega_{3} M\left(y_{\alpha i}^{+} 1_{A}^{-} 2_{B}^{-} z_{\beta}^{+} x_{j}\right) F_{q G}\left(y_{\alpha i}^{-} 3_{C}^{-} z_{\beta}^{-} x_{j}^{*}\right)=-4 \sqrt{2} y g_{s}^{2} i f^{A B C} \int \mathrm{d} \Omega_{3} \frac{[y z]^{2}\langle y 3\rangle\langle 3 z\rangle}{[y 1][12][2 z]}
$$

where we used the fact that the integral measure is invariant under $y \leftrightarrow z$ to write the two color orderings in a similar way, and that if ${ }^{A B C} / 2=\operatorname{tr}\left(T^{A}\left[T^{B}, T^{C}\right]\right)$ and $\delta_{i j} \delta_{i j}=2$. 
The integral over the phase space angles is straightforward to do and gives $\frac{1}{2}\langle 13\rangle\langle 23\rangle /[12]$. Finally, taking into account a factor of 3 for the permutations of the three different gauge bosons, we get

$$
\gamma_{3 G \leftarrow q G}=12 \frac{y g_{s}^{2}}{\left(16 \pi^{2}\right)^{2}} .
$$

This computation, compared with the one loop reverse transition in 2.3, shows explicitly that the complexity of the computations in this method does not scale strongly with the number of loops, and two-loop computations can be easier than one-loop ones.

These two Lorentz structures, $\phi^{2} F^{2}$ and $\psi \phi F$, are the only four-particle operators that mix with the three-particle structure $F^{3}$ at two loops with a single log.

\section{$4.2\langle\cdot\rangle \rightarrow\langle\cdot\rangle^{2}:$ 5-point to 4 -point}

We start by computing a transition of the type $\psi^{2} \phi^{3} \rightarrow \psi^{2} \phi F$. For concreteness we compute the renormalisation of the hypercharge dipole of the leptons $\mathcal{O}_{e B}=\bar{\ell}_{L} \sigma_{\mu \nu} e_{R} H B_{\mu \nu}+$ h.c. due to the leptonic Yukawa $\mathcal{O}_{y_{e}}=|H|^{2} \bar{\ell}_{L} e_{R} H+$ h.c., with other pairings being similar since the non-abelian part of the amplitude plays no role in the computation. The potential contributions to the $\psi^{2} \phi^{3} \rightarrow \psi^{2} \phi F$ mixing are given by

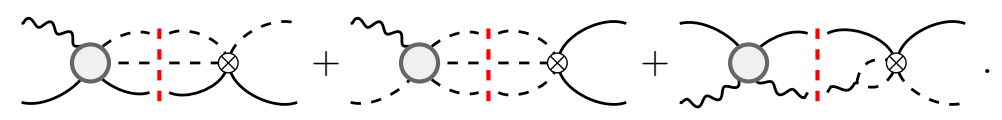

The second contribution with the three scalar in the cut is zero. This can be seen by explicit computation, but also by noticing that the fermion structure $\langle 12\rangle$ of the Yukawa is unaltered by the integration and there is no dimension-six invariant with the $F \psi^{2} \phi$ particle content and proportional to the fermion spinors $\langle 12\rangle$. The third contribution also vanishes, because the FF loop vanishes as we showed in the previous section. We are left with the tree-level amplitude contribution

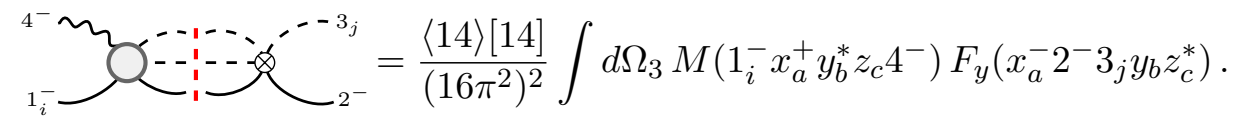

The amplitude in (4.15) is given by

$$
M\left(1_{i}^{-} 2_{j}^{+} 3_{k} 4_{l}^{*} 5^{-}\right)=-2 \sqrt{2} g^{\prime}\left(g^{\prime 2} Y_{L} Y_{H} \delta_{i j} \delta_{k l}+g^{2} T_{i j}^{A} T_{k l}^{A}\right)\left(Y_{H} \frac{[23][24]}{[12][35][45]}-Y_{L} \frac{[23][24]}{[15][25][34]}\right)
$$

where we included both $\mathrm{U}(1)_{Y}$ and $\mathrm{SU}(2)_{L}$ contributions to the amplitude, $Y_{L}$ refers to the lepton doublet and $Y_{H}$ the Higgs doublet Hypercharge. In first term in the parenthesis of (4.16) the $\mathrm{U}(1)_{Y}$ boson $5^{-}$is attached to the scalars, while in the second term the gauge boson is attached to the fermions. The FF in (4.15) is

$$
F_{y_{e}}\left(1_{i}^{-} 2^{-} 3_{j} 4_{k}^{*} 5_{l}\right)=\langle 12\rangle\left(\delta_{i j} \delta_{k l}+\delta_{i l} \delta_{k j}\right)
$$


Next, the spinors associated to the particles $x, y, z$ are rotated as in (4.2) leaving a simple trigonometric integral that gives

$$
\int \mathrm{d} \Omega_{3}\left(Y_{H} \frac{[x y][x z]\langle x 2\rangle}{[1 x][y 5][z 5]}-Y_{L} \frac{[x y][x z]\langle x 2\rangle}{[15][x 5][y z]}\right)=-Y_{H} \frac{\langle 42\rangle}{[14]}
$$

with the term proportional to the fermion hypercharge vanishing since the integrand is odd under $y \leftrightarrow z$ while the integral measure is invariant. Therefore all in all we get

$$
(4.15)=\frac{1}{\left(16 \pi^{2}\right)^{2}} g^{\prime} Y_{H}\left(g^{\prime 2} Y_{L} Y_{H}(N+1)+g^{2} \frac{N^{2}-1}{2 N}\right) 2 \sqrt{2} \delta_{i j}\langle 14\rangle\langle 42\rangle,
$$

where $N=2$ for the doublet. Exchanging $1^{-} \leftrightarrow 2^{-}$in (4.15) amounts to $Y_{L} \leftrightarrow Y_{R}$ in the order $g^{\prime 3}$ contribution. Thus all in all, we get the anomalous dimension

$$
\gamma_{e B \leftarrow y_{e}}=\frac{3}{\left(16 \pi^{2}\right)^{2}}\left(g^{\prime 3}\left(Y_{L}+Y_{R}\right) Y_{H}^{2}+g^{\prime} g^{2} Y_{H} \frac{1}{4}\right)
$$

in agreement with [46].

The second case we consider is the renormalisation of the $\phi^{2} F^{2}$ operators due to the dimension-six Yukawa $O_{y}$. For instance, take the operator to be $O_{G G}$. As before, denote by $\alpha, \beta$ the color indices and by $i, j, \ldots$ the $\mathrm{SU}(2)_{L}$ ones. There are two types of contributions, given by the diagrams

$$
\text { nt: }
$$

The one-loop FF vanishes as before, and we have excluded those one-loop FFs that are contracted with $M\left(\phi \phi \rightarrow g^{-} g^{-}\right)=0$ at tree-level. Thus we are left with the first contribution in (4.21). Both the MFF and the 5-point amplitude have already appeared and they are given in (2.19) and (2.37), respectively. The phase-space integral in (4.31) gives

$$
\int \mathrm{d} \Omega_{3} M\left(y_{\alpha k}^{+} 1_{A}^{-} 2_{B}^{-} z_{\beta}^{+} x_{l}^{*}\right) F\left(y_{\alpha k}^{-} z_{\beta j}^{-} x_{l} 3_{i} 4_{j}^{*}\right)=-y g_{s}^{2} \delta^{A B} \delta_{i j}(N+1) 2 \int \mathrm{d} \Omega_{3} \frac{[y z]^{2}\langle y z\rangle}{[y 1][12][2 z]},
$$

where the factor 2 comes from the two color orderings giving the same integrand, $\delta^{A B}=2 \operatorname{tr}\left(T^{A} T^{B}\right)$ and the $(N+1)$ factor, with $N=2$, comes from the $\mathrm{SU}(2)_{L}$ contraction. The integral over the trigonometric angles gives $-\langle 12\rangle /[12]$. The result is

$$
\gamma_{F F \leftarrow y}=-3 \frac{y g_{s}^{2}}{\left(16 \pi^{2}\right)^{2}} .
$$

The last contribution of the type $\langle\cdot\rangle \rightarrow\langle\cdot\rangle^{2}$ is the $\psi^{2} \phi^{3} \rightarrow \psi^{4}$ transition,

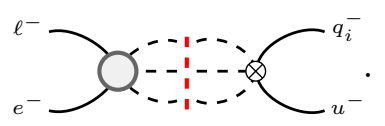


There is not a contribution from a one-loop FF. Note that the one-loop form factor where one scalar line is closed into a fermion leg vanishes on-shell because it is proportional to $\not q q$. Indeed such loops are proportional to $(\bar{q} \not \partial q)|H|^{2}+$ h.c. in an effective action calculation, see e.g. [15].

For definitenes, we compute the renormalisation of the 4-fermion operator $O_{\text {lequ }}=\epsilon^{i j}\left(\bar{\ell}_{L}^{i} e_{R}\right)\left(\bar{q}^{j} u_{R}\right)$ by the up-quark Yukawa $O_{y_{u}}=|H|^{2} \bar{q}_{L}^{i} u_{R} \epsilon_{i j} H^{j *}$ with MFF

$$
\begin{aligned}
& F_{l e q u}\left(1_{i}^{-} 2^{-} 3_{j}^{-} 4^{-}\right)=\epsilon^{i j}\langle 12\rangle\langle 34\rangle, \\
& F_{y_{u}}\left(1_{i}^{-} 2^{-} 3_{j}^{*} 4_{k} 5_{l}^{*}\right)=\left(\epsilon_{i j} \delta_{k l}+\epsilon_{i l} \delta_{k j}\right)\langle 12\rangle .
\end{aligned}
$$

Any other choice of Yukawa and 4-fermion operators will be computed in a similar fashion. The 5-point amplitude involved has three components, proportional to $y_{e}^{3}, y_{e} \lambda$ and $y_{e} g^{2}$ or $y_{e} g^{\prime 2}$. The contributions dependent on the gauge coupling are proportional to the difference of two scalar momenta, say $\left(p_{5}-p_{4}\right)_{\mu}$, since there is always a scalar current coupled to a gauge boson. Since the FF is independent of the scalar momenta, the phase space integration sets these contributions to zero. The only relevant part of the amplitude is then

$$
M\left(1_{i}^{-} 2^{-} 3_{j} 4_{k}^{*} 5_{l}\right)=-y_{e}^{3}\left(\delta_{i}^{j} \delta_{k}^{l} \frac{[35]}{[13][25]}+\delta_{i}^{l} \delta_{k}^{j} \frac{[53]}{[15][23]}\right)+2 y \lambda\left(\delta_{i}^{j} \delta_{k}^{l}+\delta_{i}^{l} \delta_{k}^{j}\right) \frac{1}{[12]} .
$$

The convolution is

$$
\begin{aligned}
& \int \mathrm{d} \Omega_{3} M\left(\ell_{i}^{-} e^{-} x_{a} y_{b}^{*} z_{c}\right) F_{y_{u}}\left(q_{j}^{-} u^{-} x_{a}^{*} y_{b} z_{c}^{*}\right) \\
&=2(N+1) \epsilon_{j i} \int \mathrm{d} \Omega_{3}\left[-y_{e}^{3} \frac{[x z]}{[\ell x][e z]}\langle q u\rangle+2 y_{e} \lambda \frac{\langle q u\rangle}{[\ell e]}\right],
\end{aligned}
$$

where we labeled the external momenta as $\ell, e, q, u$ to keep track of the fermions' flavour, and $N=2$ is the dimension of the doublet. The second trigonometric integral is trivial and gives 1 , while the first one gives $2\langle q u\rangle /[\ell e]$. After multiplying this by the phase space factor $\langle\ell e\rangle[\ell e]$ and a $1 / 2$ ! for the identical particles $x$ and $z$, we identify the resulting factor $\epsilon^{i j}\langle\ell e\rangle\langle q u\rangle$ as the one associated with the operator $O_{l e q u}=\epsilon^{i j}\left(\bar{\ell}_{L}^{i} e_{R}\right)\left(\bar{q}_{L}^{j} u_{R}\right)$, with an anomalous dimension

$$
\gamma_{l e q u \leftarrow y_{u}}=-\frac{6}{\left(16 \pi^{2}\right)^{2}}\left(y_{e} \lambda-y_{e}^{3}\right) .
$$

\section{$4.31 \rightarrow\langle\cdot\rangle: 6$-point to 5-point}

Lastly, consider the transition between the operator $O_{6}=|H|^{6}$ with MFF

$$
F_{6}\left(1_{i} 2_{j}^{*} 3_{k} 4_{l}^{*} 5_{m} 6_{n}^{*}\right)=6 \delta_{i}^{j} \delta_{k}^{l} \delta_{m}^{n}+5 \text { perm. of }\{j, l, n\}
$$

and the dimension-six Yukawa $O_{y}$. The only non-vanishing contribution comes from the diagram

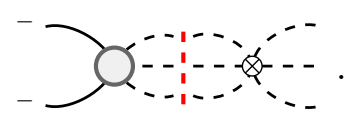


The integral over the phase space is the same we encountered in the preceding section for the $\psi^{2} \phi^{3} \rightarrow \psi^{4}$ transition. The flavour contraction gives $2(N+2)\left(\delta_{i}^{j} \delta_{k}^{l}+\delta_{i}^{l} \delta_{k}^{j}\right)$. Thus we are led to

$$
\gamma_{y_{e} \leftarrow 6}=\frac{48}{\left(16 \pi^{2}\right)^{2}}\left(y_{e} \lambda-y_{e}^{3}\right) .
$$

\section{Conclusions}

In this work we have demonstrated how to retrieve the anomalous dimensions of the SM EFT operators using the on-shell S-matrix and form factors. In particular we showed how only tree-level S-matrix elements and FFs can be used to determine all of the one-loop and many two-loop anomalous dimensions of the SM EFT. Another nice aspect of this method is that it re-uses the same amplitudes to determine many anomalous dimensions. For instance, the amplitude $M(F F \phi \psi \psi)$ appears in the calculation of the one loop $F^{3} \rightarrow \psi^{2} \phi F$, $\phi^{2} F^{2} \rightarrow \phi^{3} \psi^{2}$, and the two loop $\psi^{2} \phi^{3} \rightarrow \phi^{2} F^{2}$ and $\psi^{2} \phi F \rightarrow F^{3}$ mixings. This aspect of the method permits many self-consistency checks. Our calculations could be fully automated and extended to the entire one-loop anomalous dimension matrix of the leading higher dimensional operators in the SM EFT, or a more general field theory.

In section 2 we computed a variety of representative one-loop anomalous dimensions including the order $\lambda$ corrections, examples involving IR anomalous dimensions and nonminimal form factors. In section 3 we have discussed the structure of the one-loop anomalous dimension matrix of dimension-six operators. This has allowed us to identify the most interesting two-loop anomalous dimensions, namely those that are forbidden at one-loop either by leg counting or by helicity selection rules only. In section 4 we have computed the two-loop anomalous dimensions that mix operators as indicated by the blue arrows in figure 1 as an illustration of the power of the on-shell method.

Current and future precision experiments probe and will probe the RG structure at two loops. The paradigmatic example is the measurement of the electron EDM [46, 47], whose current bound tests multi-TeV dynamics at two loops. Other future experiments, like the measurements of the $\operatorname{Br}(\mu \rightarrow e \gamma)$ at the MEG-II experiment will reach a precision of $6 \cdot 10^{-14}$ [48]. This would translate into a bound of $\Lambda \gtrsim 10^{3} \mathrm{TeV}$ for the operator $\mathcal{L} \supset \frac{\sqrt{y_{e} y_{\mu}}}{\Lambda^{2}} \bar{\mu} \sigma_{\mu \nu} e H F_{\mu \nu}$. If the UV dynamics is such that, instead of the dipole, an operator $\frac{\sqrt{y_{e} y_{\mu}}}{\Lambda_{i}^{2}} O_{i}$, which only affects the dipole at two loop order, is generated at the high scale, then the log-enhanced contribution due to the two-loop RG mixing would set a constraint of $\Lambda \gtrsim 10 \mathrm{TeV}$. Having a complete map of the two-loop anomalous dimensions of the operators probed by this and other future precision experiments, will provide the complete characterisation of the dynamics affecting such processes through a log-enhanced contribution.

While we have shown how to compute the transitions $\langle\cdot\rangle^{2} \rightarrow\langle\cdot\rangle^{3},\langle\cdot\rangle \rightarrow\langle\cdot\rangle^{2}$ and $1 \rightarrow\langle\cdot\rangle$, it will be interesting to compute the rest of the two-loop anomalous dimensions for which the corresponding one-loop contributions are absent, as well as those for which the one-loop contribution are suppressed by a small coupling, like a Yukawa of a light particle. In addition, we look forward to investigating other aspects of this method. Whether we 
can relate the branch-cut discontinuity of other physical quantities to the Callan-Symanzik equation using the dilatation operator (1.4), or compute certain anomalous dimensions at large orders, are some of the questions that we are eager to investigate further.

Note added. Simultaneously with the submission of this preprint ref. [49] appeared where the renormalisation of higher-dimensional operators from on-shell amplitudes is presented from a slightly different perspective. In ref. [49] all one-loop anomalous dimensions of the $\mathrm{SU}(2)_{L}$ dipole operator of the electron (up to self-renormalisation) are reproduced.

Shortly after, ref. [50] appeared where the computation of SM EFT operator anomalous dimensions using unitarity cuts is also discussed and the self-renormalisation of $|H|^{2} B_{\mu \nu}^{2}$ and $G_{\mu \nu}^{3}$ is reproduced. There instances of applying these techniques to operators of dimension larger than six are also discussed.

Before we submitted to the journal, ref. [51] appeared where the use of on-shell methods to compute one and two-loop anomalous dimensions in the context of EFTs containing higher-dimension operators is described. The pattern of vanishing entries in the two-loop anomalous dimension matrix is also analysed.

\section{Acknowledgments}

JEM and JI are grateful to the participants of the SISSA-ICTP joint Journal Club for useful discussions on this topic. MR acknowledges funding from the Swiss National Science Foundation under grant no. PP002-170578.

\section{A Phase space integrals}

We are interested in evaluating " $n \rightarrow 2$ " integrals of the type

$$
I_{n \rightarrow 2}=\int \frac{1}{n !} \prod_{i=1}^{n} \frac{d^{3} p_{i}^{\prime}}{(2 \pi)^{3} 2 p_{i}^{\prime 0}}(2 \pi)^{4} \delta^{4}\left(p_{1}+p_{2}-\sum_{i=1}^{n} p_{i}^{\prime}\right)\left\langle 12\left|M_{n \rightarrow 2}\right| 1^{\prime} \ldots n^{\prime}\right\rangle\left\langle 1^{\prime} \ldots n^{\prime}|\mathcal{O}| 0\right\rangle
$$

These integrals feature an S-matrix element with $n$ particles in the initial state and 2 in the final state. We include a symmetry factor $1 / n$ ! for identical particles.

For massless particles, the integrand is expressed more conveniently in terms of spinor helicity variables $\lambda_{i}^{\prime}$, rather than the momenta. Assuming that $s_{12}=\langle 12\rangle[21]$ is nonzero, the two spinors corresponding to the external particles $\lambda_{1}$ and $\lambda_{2}$ are linearly independent, and form a complete basis. Therefore the spinors for the $n$ exchanged particles (whose momenta are integrated over) may be expressed as

$$
\left(\begin{array}{c}
\lambda_{1}^{\prime} \\
\lambda_{2}^{\prime} \\
\vdots \\
\lambda_{n}^{\prime}
\end{array}\right)=A\left(\begin{array}{c}
\lambda_{1} \\
\lambda_{2}
\end{array}\right), \quad A=\left(\begin{array}{cc}
a_{11} & a_{12} \\
a_{21} & a_{22} \\
\vdots & \vdots \\
a_{n 1} & a_{n 2}
\end{array}\right),
$$


where the $a_{i b}$ are arbitrary complex numbers. Next we recast the measure and momentum conserving delta function according to the change of variables in (A.2).

The momentum conserving delta function is then given by

$$
\delta^{4}(P)=4 \delta\left(\lambda^{1} \bar{\lambda}^{\dot{1}}\right) \delta\left(\lambda^{1} \bar{\lambda}^{\dot{2}}\right) \delta\left(\lambda^{2} \bar{\lambda}^{\dot{1}}\right) \delta\left(\lambda^{2} \bar{\lambda}^{\dot{2}}\right)=\frac{4}{\langle 12\rangle^{2}[12]^{2}} \delta\left(u_{11}\right) \delta\left(u_{12}\right) \delta\left(u_{21}\right) \delta\left(u_{22}\right),
$$

where $P^{\alpha \dot{\alpha}} \equiv \lambda^{\alpha} \bar{\lambda}^{\dot{\alpha}} \equiv \lambda_{1}^{\alpha} \bar{\lambda}_{1}^{\dot{\alpha}}+\lambda_{2}^{\alpha} \bar{\lambda}_{2}^{\dot{\alpha}}-\sum_{i=1}^{n} \lambda_{i}^{\prime \alpha} \bar{\lambda}_{i}^{\prime \dot{\alpha}}$. And we have defined the $2 \times 2$ matrix $u_{n m}=\delta_{n m}-\left(A^{\dagger} A\right)_{n m}$. Thus, the momentum conserving delta function imposes the constraint $A^{\dagger} A=\mathbb{1}_{2}$.

Now we consider the measure for one of the $n$ exchanged particles. We would like to express it in terms of the $a_{i b}$ variables, perhaps like:

$$
\frac{d^{3} p_{i}^{\prime}}{(2 \pi)^{3} 2 p_{i}^{\prime 0}} \stackrel{?}{=} f\left(a_{i 1}, a_{i 2}\right) d a_{i 1} d a_{i 1}^{*} d a_{i 2} d a_{i 2}^{*}
$$

This measure only depends on $a_{i 1}$ and $a_{i 2}$ because the measure depends only upon $p_{i}^{\prime \mu}$ and $p_{i}^{\prime \mu} \sigma_{\mu}^{\alpha \dot{\alpha}}=\lambda_{i}^{\prime \alpha} \bar{\lambda}_{i}^{\prime \dot{\alpha}}$. However, there are three real variables to be integrated over on the l.h.s. but four on the r.h.s., so the relationship above cannot be true. The problem arises due to a redundant phase that emerges when the kinematic information is expressed in the $a_{i b}$ variables rather than in the momenta. To be specific, we can rotate complex $a_{i 1}$ and $a_{i 2}$ by a common phase so that

$$
a_{i 1} \rightarrow e^{i \alpha_{i}} a_{i 1}, \quad a_{i 2} \rightarrow e^{i \alpha_{i}} a_{i 2}, \quad \lambda_{i}^{\prime} \rightarrow e^{i \alpha_{i}} \lambda_{i}^{\prime} .
$$

This rotation leaves $p_{i}^{\prime \alpha \dot{\alpha}}=\lambda_{i}^{\prime \alpha} \bar{\lambda}_{i}^{\prime \dot{\alpha}}$ unchanged, so clearly the measure, and the momentum conserving delta function are invariant under this rotation. Equally, the integrand is also independent of this global phase

$$
\left\langle 12\left|\mathcal{M}_{n \rightarrow 2}\right| 1^{\prime} \ldots n^{\prime}\right\rangle\left\langle 1^{\prime} \ldots n^{\prime}|\mathcal{O}| 0\right\rangle \rightarrow e^{2 i h_{i} \alpha_{i}}\left\langle 12\left|\mathcal{M}_{n \rightarrow 2}\right| 1^{\prime} \ldots n^{\prime}\right\rangle \cdot e^{-2 i h_{i} \alpha_{i}}\left\langle 1^{\prime} \ldots n^{\prime}|\mathcal{O}| 0\right\rangle,
$$

where $h_{i}$ is the helicity of particle $i$. All quantities in the integral are therefore invariant under the global phase transformation, so that adjusting it is analogous to performing a gauge transformation in a gauge theory. Just as in gauge theory, it is convenient to fix a value for this "gauge parameter". This can be done in a way that still leaves the equivalence of different gauge fixing conditions manifest using the Fadeev-Popov trick: we multiply the measure by the following factor

$$
\int d \alpha_{i} \delta\left(f_{i}\left(\alpha_{i}\right)\right) f_{i}^{\prime}\left(\alpha_{i}\right)=1
$$

Defining the overall phase to be $\alpha_{i} \equiv \arg \left(a_{i 1}\right)+\arg \left(a_{i 2}\right)$, the measure becomes

$$
\frac{d^{3} p_{i}^{\prime} d \theta_{i}}{(2 \pi)^{3} 2 p_{i}^{\prime 0}}=\frac{\langle 12\rangle[21]}{2(2 \pi)^{3}} d a_{i 1} d a_{i 1}^{*} d a_{i 2} d a_{i 2}^{*} .
$$

Putting everything back together, the original integral can be written as

$$
\begin{aligned}
I_{n \rightarrow 2}=\frac{(\langle 12\rangle[21])^{n-2}}{2^{n-2} n !(2 \pi)^{3 n-4}} \int \prod_{i=1}^{n}\left[d^{2} a_{i 1} d^{2} a_{i 2}\right. & \left.\delta\left(f_{i}\left(\alpha_{i}\right)\right) f_{i}^{\prime}\left(\alpha_{i}\right)\right] \delta^{4}\left(A^{\dagger} A-\mathbb{1}_{2}\right) \\
& \times\left\langle 12\left|\mathcal{M}_{n \rightarrow 2}\right| 1^{\prime} \ldots n^{\prime}\right\rangle\left\langle 1^{\prime} \ldots n^{\prime}|\mathcal{O}| 0\right\rangle .
\end{aligned}
$$




\section{A.1 The $2 \rightarrow 2$ case}

This is the simplest case. We would like to parametrize the matrix $A$ so that the delta function constraints take a particularly simple form. This can be achieved with a polar decomposition

$$
A=U P
$$

where $U$ is a unitary $2 \times 2$ matrix and $P$ is a positive-semidefinite hermitian matrix. Without loss of generality we parametrise $P$ and $U$ as

$$
P=\left(\begin{array}{cc}
r & u+i v \\
u-i v & t
\end{array}\right), \quad U=\left(\begin{array}{cc}
e^{i \rho} \cos \theta & -e^{i \rho} \sin \theta e^{i \phi} \\
e^{i \eta} \sin \theta e^{-i \phi} & e^{i \eta} \cos \theta
\end{array}\right)
$$

where $r, u, v, t$ are real valued and the angles have the ranges $0 \leqslant \rho, \eta, \phi<2 \pi$ and $0 \leqslant \theta<\pi / 2$. The delta function constraint acts purely on the hermitian matrix (A.10)

$$
\delta^{4}\left(A^{\dagger} A-\mathbb{1}_{2}\right)=\frac{1}{32} \delta(r-1) \delta(u) \delta(v) \delta(t-1) .
$$

Next, using (A.11) and fixing $P=\mathbb{1}_{2}$, the delta function constraints that eliminate gauge redundancy take a simpler form $\theta_{1}=2 \rho+\phi, \quad \theta_{2}=2 \eta-\phi$. We can choose any topologically equivalent functions for the $f_{i}\left(\alpha_{i}\right)$, but it is convenient to pick the gauge where $f_{1}\left(\alpha_{1}\right)=\frac{1}{2}\left(\alpha_{1}-\phi\right)$ and $f_{2}\left(\alpha_{2}\right)=\frac{1}{2}\left(\alpha_{2}+\phi\right)$. The gauge fixing delta functions become $\delta\left(f_{1}\left(\alpha_{1}\right)\right) f_{1}^{\prime}\left(\alpha_{1}\right) \delta\left(f_{2}\left(\alpha_{2}\right)\right) f_{2}^{\prime}\left(\alpha_{2}\right)=\frac{1}{4} \delta(\rho) \delta(\eta)$. All in all, in terms of the new parameters, the integral measure becomes

$$
d^{2} a_{11} d^{2} a_{12} d^{2} a_{21} d^{2} a_{22}=32 \sin 2 \theta d r d u d v d t d \rho d \eta d \theta d \phi
$$

once we have set $P=\mathbb{1}_{2}$. Putting everything together, the $2 \rightarrow 2$ integral becomes

$$
I_{2 \rightarrow 2}=\frac{1}{16 \pi} \int_{0}^{2 \pi} \frac{d \phi}{2 \pi} \int_{0}^{\pi / 2} 2 \sin \theta \cos \theta d \theta\left\langle 12\left|\mathcal{M}_{2 \rightarrow 2}\right| 1^{\prime} 2^{\prime}\right\rangle\left\langle 1^{\prime} 2^{\prime}|\mathcal{O}| 0\right\rangle
$$

\section{A.2 The $3 \rightarrow 2$ case}

Here we consider the phase space integral when 3 particles are exchanged across the cut. The calculation of the integral measure and transformation law for the spinors representing the exchanged particles proceeds in a similar way to the 2 particle case; the delta function constraints take a simpler form when the complex $3 \times 2$ matrix $A$ is first expressed using a singular value decomposition, analogous to a polar decomposition for matrices that are not square

$$
A=U \Sigma V^{\dagger}
$$

$U$ is a unitary $3 \times 3$ matrix, $\Sigma$ is a $3 \times 2$ rectangular diagonal matrix with real, non-negative entries, and $V$ is a unitary $2 \times 2$ matrix. This decomposition is not unique however. It can be seen that applying the following transformation leaves $A$ unchanged:

$$
U \rightarrow U \operatorname{diag}\left\{e^{i \phi_{a}}, e^{i \phi_{b}}, e^{i \phi_{c}}\right\}, \quad V \rightarrow V \operatorname{diag}\left\{e^{i \phi_{a}}, e^{i \phi_{b}}\right\} .
$$


To eliminate this redundancy, we choose to fix two of the undetermined phases in $V$ and one in $U$. With these restrictions, $U$ can be parametrized as

$$
U=\operatorname{diag}\left\{e^{i \phi_{2}}, e^{i \phi_{3}}, e^{i \phi_{4}}\right\}\left(\begin{array}{ccc}
c_{1} & -s_{1} c_{3} & -s_{1} s_{3} \\
s_{1} c_{2} & c_{1} c_{2} c_{3}-s_{2} s_{3} e^{i \delta} & c_{1} c_{2} s_{3}+s_{2} c_{3} e^{i \delta} \\
s_{1} s_{2} & c_{1} s_{2} c_{3}+c_{2} s_{3} e^{i \delta} & c_{1} s_{2} s_{3}-c_{2} c_{3} e^{i \delta}
\end{array}\right) \operatorname{diag}\left\{1, e^{i \phi}, 1\right\},
$$

where $c_{i} \equiv \cos \theta_{i}$ and $s_{i} \equiv \sin \theta_{i}$. Note that alternative parametrizations of an arbitrary $3 \times 3$ unitary matrix can be found by taking any parametrization of the CKM matrix and multiplying on either side by diagonal matrices of phases. We parametrize $\Sigma$ and $V$ (unitary matrix minus two redundant phases) as

$$
\Sigma=\left(\begin{array}{ll}
r & 0 \\
0 & t \\
0 & 0
\end{array}\right), \quad V=\left(\begin{array}{cc}
c_{4} & -s_{4} e^{i \phi_{5}} \\
s_{4} e^{-i \phi_{5}} & c_{4}
\end{array}\right)
$$

In each case, the allowed ranges for the parameters is $0 \leqslant \theta_{i}<\pi / 2,0 \leqslant r, t<\infty$ and $0 \leqslant \phi_{i}<2 \pi$. The delta function constraints depend only upon the parameters in $\Sigma$ and $V$ :

$$
\delta\left(A^{\dagger} A-\mathbb{1}_{2}\right)=\frac{1}{8 r t\left(r^{2}-t^{2}\right)^{2} s_{4} c_{4}} \delta(r-1) \delta(t-1) \delta\left(\theta_{4}\right) \delta\left(\phi_{5}\right) .
$$

The denominator is zero at the point in parameter space selected by the delta functions. This is just a harmless coordinate singularity though, which gets removed when combined with the integral measure in these new variables. This measure is

$$
\prod_{i=1}^{3}\left[d^{2} a_{i 1} d^{2} a_{i 2}\right]=64 r^{3} t^{3}\left(r^{2}-t^{2}\right)^{2} s_{1}^{3} c_{1} s_{2} c_{2} s_{3} c_{3} s_{4} c_{4} \prod_{j=1}^{4} d \theta_{j} \prod_{k=1}^{5} d \phi_{k} d r d t d \delta .
$$

Similarly to the $2 \rightarrow 2$ case, the gauge fixing delta functions become

$$
\delta\left(f_{1}\left(\alpha_{1}\right)\right) f_{1}^{\prime}\left(\alpha_{1}\right) \delta\left(f_{2}\left(\alpha_{2}\right)\right) f_{2}^{\prime}\left(\alpha_{2}\right) \delta\left(f_{3}\left(\alpha_{3}\right)\right) f_{3}^{\prime}\left(\alpha_{3}\right)=\frac{1}{8} \delta\left(\phi_{2}\right) \delta\left(\phi_{3}\right) \delta\left(\phi_{4}\right) .
$$

Putting everything together into (A.9), yields the following result for the $I_{3 \rightarrow 2}$ integral

$$
I_{3 \rightarrow 2}=\frac{\langle 12\rangle[21]}{4^{4} \pi^{3} 3 !} \int d \Omega_{3}\left\langle 12\left|\mathcal{M}_{3 \rightarrow 2}\right| 1^{\prime} 2^{\prime} 3^{\prime}\right\rangle\left\langle 1^{\prime} 2^{\prime} 3^{\prime}|\mathcal{O}| 0\right\rangle
$$

with the measure given by

$$
d \Omega_{3}=4 \cos \theta_{1} \sin ^{3} \theta_{1} d \theta_{1} 2 \cos \theta_{2} \sin \theta_{2} d \theta_{2} 2 \cos \theta_{3} \sin \theta_{3} d \theta_{3} \frac{d \delta}{2 \pi} \frac{d \phi}{2 \pi} .
$$

After the all of the constraints have been applied, the transformation law for the spinors is

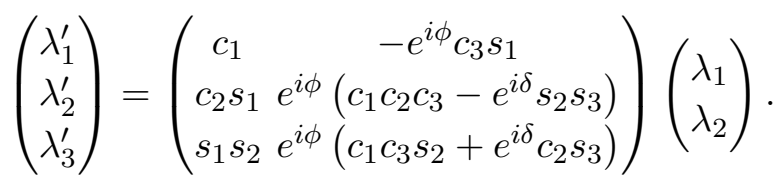




\section{B Amplitudes}

We computed some high point amplitudes of the text using the BCFW technique [52]. ${ }^{13}$ In this appendix we illustrate how to compute the five point amplitude in (2.37). An advantage of using BCFW w.r.t. Feynman diagrams is that it automatically produces nice compact expressions that simplify our phase-space integrations.

To apply BCFW recursion relations we need the lower order seeds, namely the three point amplitudes. The three point amplitude between two fermions and a gluon is ${ }^{14}$

$$
M\left(1_{i}^{-} 2_{j}^{+} 3_{A}^{-}\right)=-g \sqrt{2} T_{i j}^{A} \frac{\langle 13\rangle^{2}}{\langle 12\rangle} \text { and } M\left(1_{i}^{-} 2_{j}^{+} 3_{A}^{+}\right)=-g \sqrt{2} T_{i j}^{A} \frac{[13]^{2}}{[12]},
$$

and for a Yukawa interaction $\mathcal{L} \supset-y H \bar{q}_{L} q_{R}$,

$$
M\left(1^{-} 2^{-} 3\right)=-y\langle 12\rangle \text { and } M\left(1^{+} 2^{+} 3\right)=-y[12] .
$$

In this case we do not need the three point amplitude between two scalars and a gauge boson because the Higgs does not carry color.

To compute the five point amplitude (2.37) we need the 4-point amplitude between two same-helicity fermions, a scalar and a gluon. It is easy to do it with the usual techniques, but we will do it using the BCFW recursion relation. See [12] for a review.

BCFW proceeds by shifting the spinors by a complex parameter $z$ so that the shifted momenta $\hat{p}$ is on-shell and total momentum is preserved, $\hat{p}^{2}=0$ and $\sum_{i} p_{i}^{\mu}=0$. For instance, in a $n$-particle amplitude a $[1,2\rangle$-shift consists on shifting the spinors of $p_{1}$ and $p_{2}$ as $\left.\left.\left.\left.\left.\mid \hat{1}\right]=\mid 1\right]+z \mid 2\right], \mid \hat{2}\right]=\mid 2\right],|\hat{1}\rangle=|1\rangle,|\hat{2}\rangle=|2\rangle-z|1\rangle$, and one can check that this preserves momentum conservation of on-shell momenta. For instance, applying this shift on the $M\left(1_{i}^{-} 2_{A}^{ \pm} 3_{j}^{-} 4\right)$ amplitude, BCFW splits it into two on-shell 3-point amplitudes

$$
\overbrace{\hat{1}_{i}^{-}}^{3_{\hat{2}_{A}^{ \pm}}^{-}}=M_{L}\left(\hat{1}_{i}^{-} \hat{p}_{k}^{-} 4\right) \frac{1}{\langle 14\rangle[14]} M_{R}\left(-\hat{p}_{k}^{-} 3_{j}^{-} \hat{2}_{A}^{ \pm}\right) \text {. }
$$

There are two different cases depending on the helicity of the gluon. If the gluon has negative helicity, the numerator of the right amplitude is $\langle 3 \hat{2}\rangle^{2}$. However, this is evaluated at a $\hat{p}^{2}=0=\langle\hat{2} 3\rangle[\hat{2} 3]=\langle\hat{2} 3\rangle[23]$. This means that $z$ is such that $\langle\hat{2} 3\rangle=0$, and therefore we recover that the all-minus amplitude vanishes $M\left(1_{i}^{-} 2_{A}^{-} 3_{j}^{-} 4\right)=0$. Instead, if the gluon has positive helicity we get,

$$
M\left(1_{i}^{-} 2_{A}^{+} 3_{j}^{-} 4\right)=y g \sqrt{2} T_{i j}^{A}\langle\hat{1} \hat{p}\rangle \frac{1}{\langle 14\rangle[14]} \frac{[\hat{p} \hat{2}]^{2}}{[\hat{p} 3]},
$$

where we used $\hat{p}=-\hat{p}_{1}-p_{4},\langle 1 \hat{p}\rangle[\hat{p} 2]=-\langle 14\rangle[42]$. A basic trick to get rid of $\hat{p}$ is to multiply the amplitude by $\langle 2 \hat{p}\rangle /\langle 2 \hat{p}\rangle$, and then write $\langle 2 \hat{p}\rangle[\hat{p} 2]=\langle 23\rangle[32]$ and $\langle 2 \hat{p}\rangle[\hat{p} 3]=\langle 2 \hat{2}\rangle[23]$. As we showed, this last factor has to be evaluated at $\langle\hat{2} 3\rangle=0=\langle 23\rangle-z\langle 13\rangle$ so that

\footnotetext{
${ }^{13}$ Note that some of the SM amplitudes have a pole at infinity in the BCFW complex plane. In those cases we used standard Feynman diagram calculation.

${ }^{14}$ The normalisation is such that the covariant derivative is $D_{\mu}=\partial_{\mu}+i T_{i j}^{A} G_{\mu}^{A}$.
} 
$z=-\langle 23\rangle /\langle 13\rangle$. At this value, $\langle 2 \hat{2}\rangle=-z\langle 12\rangle=\langle 12\rangle\langle 23\rangle /\langle 13\rangle$. Then putting everything together, we get

$$
M\left(1_{i}^{-} 2_{A}^{+} 3_{j}^{-} 4\right)=-y g \sqrt{2} T_{i j}^{A} \frac{[24]^{2}}{[14][34]}=y g \sqrt{2} T_{i j}^{A} \frac{\langle 13\rangle^{2}}{\langle 12\rangle\langle 23\rangle} .
$$

Next we use the 4-point amplitude in (B.5) to construct the 5-point amplitude $M\left(1_{i}^{-} 2_{A}^{+} 3_{B}^{+} 4_{j}^{-} 5\right)$. We do a $[2,3\rangle$-shift. There are two contributions,

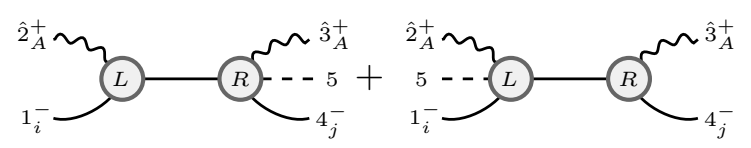

the two diagrams are given by

$$
M_{L}\left(1_{i}^{-} \hat{p}_{k}^{+} \hat{2}_{A}^{+}\right) \frac{1}{p_{12}^{2}} M_{R}\left(-\hat{p}_{k}^{-} \hat{3}_{B}^{+} 4_{j}^{-} 5\right)+M_{L}\left(1_{i}^{-} \hat{2}_{A}^{+} \hat{p}_{k}^{-} 5\right) \frac{1}{p_{34}^{2}} M_{R}\left(-\hat{p}_{k}^{+} \hat{3}_{B}^{+} 4_{j}^{-}\right)
$$

where we have fixed a particular color ordering, namely $\left(T^{A} T^{B}\right)_{i j}$. Exchanging $2 \leftrightarrow 3$ in the sub-amplitudes generates the other color ordering $\left(T^{B} T^{A}\right)_{i j}$. The first diagram vanishes because it is evaluated at $[1 \hat{2}]=0$, and $|\hat{p}\rangle[\hat{p} \hat{2}]=\left(p_{1}+\hat{p}_{2}\right)|\hat{2}\rangle=-|1\rangle[1 \hat{2}]=0$. Hence $[\hat{p} \hat{2}]=0$ as well, and therefore the left amplitude vanishes. The second diagram gives

$$
M\left(1_{i}^{-} 2_{A}^{+} 3_{B}^{+} 4_{j}^{-} 5\right)=-2 y g^{2}\left(T^{A} T^{B}\right)_{i j} \frac{\langle 1 \hat{p}\rangle^{2}}{\langle 1 \hat{2}\rangle\langle\hat{2} \hat{p}\rangle} \frac{1}{\langle 34\rangle[34]} \frac{[\hat{p} \hat{3}]^{2}}{[\hat{p} 4]} .
$$

The same argument does not work for this diagram, since the right amplitude is proportional to $[\hat{p} \hat{3}]$ but instead it is evaluated at $\langle\hat{3} 4\rangle=0$. Now, it is straightforward to simplify the expression using $\langle 1 \hat{p}\rangle[\hat{p} \hat{3}]=\langle 14\rangle[43]$ and $\langle\hat{2} \hat{p}\rangle[\hat{p} 4]=\langle 2 \hat{3}\rangle[34]$; also, note that $\langle 2 \hat{3}\rangle=\langle 23\rangle$. Therefore the 5-point amplitude is

$$
M\left(1_{i}^{-} 2_{A}^{+} 3_{B}^{+} 4_{j}^{-} 5\right)=-2 y g^{2}\left(T^{A} T^{B}\right)_{i j} \frac{\langle 14\rangle^{2}}{\langle 12\rangle\langle 23\rangle\langle 34\rangle}-2 y g^{2}\left(T^{B} T^{A}\right)_{i j} \frac{\langle 14\rangle^{2}}{\langle 13\rangle\langle 32\rangle\langle 24\rangle} .
$$

The amplitude can be crosschecked versus the $N=4$ SYM Parke-Taylor, up to color factors.

Open Access. This article is distributed under the terms of the Creative Commons Attribution License (CC-BY 4.0), which permits any use, distribution and reproduction in any medium, provided the original author(s) and source are credited.

\section{References}

[1] CMS collaboration, Observation of a New Boson at a Mass of $125 \mathrm{GeV}$ with the CMS Experiment at the LHC, Phys. Lett. B $\mathbf{7 1 6}$ (2012) 30 [arXiv:1207.7235] [InSPIRE].

[2] ATLAS collaboration, Observation of a new particle in the search for the Standard Model Higgs boson with the ATLAS detector at the LHC, Phys. Lett. B 716 (2012) 1 [arXiv: 1207.7214] [INSPIRE]. 
[3] S. Caron-Huot and M. Wilhelm, Renormalization group coefficients and the S-matrix, JHEP 12 (2016) 010 [arXiv: 1607.06448] [INSPIRE].

[4] M. Karowski and P. Weisz, Exact Form-Factors in $(1+1)$-Dimensional Field Theoretic Models with Soliton Behavior, Nucl. Phys. B 139 (1978) 455 [InSPIRE].

[5] K.M. Watson, The Effect of final state interactions on reaction cross-sections, Phys. Rev. 88 (1952) 1163 [INSPIRE].

[6] K.M. Watson, Some general relations between the photoproduction and scattering of $\pi$ mesons, Phys. Rev. 95 (1954) 228 [inSPIRE].

[7] S. Weinberg, The Quantum theory of fields. Vol. 1: Foundations, Cambridge University Press (2005) [INSPIRE].

[8] F.A. Berends, R. Kleiss, P. De Causmaecker, R. Gastmans and T.T. Wu, Single Bremsstrahlung Processes in Gauge Theories, Phys. Lett. B 103 (1981) 124 [InSPIRE].

[9] F.A. Berends, R. Kleiss, P. De Causmaecker, R. Gastmans, W. Troost and T.T. Wu, Multiple Bremsstrahlung in Gauge Theories at High-Energies. 2. Single Bremsstrahlung, Nucl. Phys. B 206 (1982) 61.

[10] R. Kleiss and W. Stirling, Spinor Techniques for Calculating p $\bar{p} \rightarrow W_{ \pm} / Z_{0}+$ Jets, Nucl. Phys. B 262 (1985) 235 [INSPIRE].

[11] J.M. Henn and J.C. Plefka, Scattering Amplitudes in Gauge Theories, vol. 883, Springer, Berlin (2014) [DOI] [INSPIRE].

[12] H. Elvang and Y.-t. Huang, Scattering Amplitudes in Gauge Theory and Gravity, Cambridge University Press (2015) [INSPIRE].

[13] B.I. Zwiebel, From Scattering Amplitudes to the Dilatation Generator in $N=4 S Y M$, J. Phys. A 45 (2012) 115401 [arXiv:1111.0083] [InSPIRE].

[14] C. Grojean, E.E. Jenkins, A.V. Manohar and M. Trott, Renormalization Group Scaling of Higgs Operators and $\Gamma(h \rightarrow \gamma \gamma)$, JHEP 04 (2013) 016 [arXiv: 1301.2588] [INSPIRE].

[15] J. Elias-Miro, J.R. Espinosa, E. Masso and A. Pomarol, Higgs windows to new physics through $d=6$ operators: constraints and one-loop anomalous dimensions, JHEP 11 (2013) 066 [arXiv: 1308.1879] [INSPIRE].

[16] E.E. Jenkins, A.V. Manohar and M. Trott, Renormalization Group Evolution of the Standard Model Dimension Six Operators I: Formalism and lambda Dependence, JHEP 10 (2013) 087 [arXiv: 1308.2627] [INSPIRE].

[17] B. Grzadkowski, M. Iskrzynski, M. Misiak and J. Rosiek, Dimension-Six Terms in the Standard Model Lagrangian, JHEP 10 (2010) 085 [arXiv:1008.4884] [INSPIRE].

[18] R. Aoude and C.S. Machado, The Rise of SMEFT On-shell Amplitudes, JHEP 12 (2019) 058 [arXiv: 1905.11433] [INSPIRE].

[19] G. Durieux, T. Kitahara, Y. Shadmi and Y. Weiss, The electroweak effective field theory from on-shell amplitudes, JHEP 01 (2020) 119 [arXiv:1909.10551] [INSPIRE].

[20] A. Falkowski, Bases of massless EFTs via momentum twistors, arXiv:1912.07865 [INSPIRE].

[21] G. Durieux and C.S. Machado, Enumerating higher-dimensional operators with on-shell amplitudes, Phys. Rev. D 101 (2020) 095021 [arXiv: 1912.08827] [INSPIRE]. 
[22] T. Ma, J. Shu and M.-L. Xiao, Standard Model Effective Field Theory from On-shell Amplitudes, arXiv:1902.06752 [INSPIRE].

[23] T. Becher and M. Neubert, Infrared singularities of scattering amplitudes in perturbative QCD, Phys. Rev. Lett. 102 (2009) 162001 [Erratum ibid. 111 (2013) 199905] [arXiv:0901.0722] [INSPIRE].

[24] T. Becher, A. Broggio and A. Ferroglia, Introduction to Soft-Collinear Effective Theory, vol. 896, Springer (2015), [DOI] [arXiv:1410.1892] [INSPIRE].

[25] J.-y. Chiu, A. Fuhrer, R. Kelley and A.V. Manohar, Factorization Structure of Gauge Theory Amplitudes and Application to Hard Scattering Processes at the LHC, Phys. Rev. D 80 (2009) 094013 [arXiv:0909.0012] [INSPIRE].

[26] J.C. Collins, A.V. Manohar and M.B. Wise, Renormalization of the vector current in QED, Phys. Rev. D 73 (2006) 105019 [hep-th/0512187] [INSPIRE].

[27] R. Alonso, E.E. Jenkins, A.V. Manohar and M. Trott, Renormalization Group Evolution of the Standard Model Dimension Six Operators III: Gauge Coupling Dependence and Phenomenology, JHEP 04 (2014) 159 [arXiv:1312.2014] [INSPIRE].

[28] R. Barbieri, B. Bellazzini, V.S. Rychkov and A. Varagnolo, The Higgs boson from an extended symmetry, Phys. Rev. D 76 (2007) 115008 [arXiv:0706.0432] [INSPIRE].

[29] E. Braaten, C.-S. Li and T.-C. Yuan, The Evolution of Weinberg's Gluonic CP Violation Operator, Phys. Rev. Lett. 64 (1990) 1709 [INSPIRE].

[30] C. Cheung and C.-H. Shen, Nonrenormalization Theorems without Supersymmetry, Phys. Rev. Lett. 115 (2015) 071601 [arXiv: 1505.01844] [INSPIRE].

[31] B. Henning and T. Melia, Constructing effective field theories via their harmonics, Phys. Rev. D 100 (2019) 016015 [arXiv: 1902.06754] [INSPIRE].

[32] Z. Bern, J. Parra-Martinez and E. Sawyer, Nonrenormalization and Operator Mixing via On-Shell Methods, Phys. Rev. Lett. 124 (2020) 051601 [arXiv:1910.05831] [InSPIRE].

[33] A. Azatov, R. Contino, C.S. Machado and F. Riva, Helicity selection rules and noninterference for BSM amplitudes, Phys. Rev. D 95 (2017) 065014 [arXiv:1607.05236] [INSPIRE].

[34] R. Alonso, E.E. Jenkins and A.V. Manohar, Holomorphy without Supersymmetry in the Standard Model Effective Field Theory, Phys. Lett. B 739 (2014) 95 [arXiv:1409.0868] [INSPIRE].

[35] J. Elias-Miro, J.R. Espinosa and A. Pomarol, One-loop non-renormalization results in EFTs, Phys. Lett. B $\mathbf{7 4 7}$ (2015) 272 [arXiv:1412.7151] [INSPIRE].

[36] N. Craig, M. Jiang, Y.-Y. Li and D. Sutherland, Loops and Trees in Generic EFTs, JHEP 08 (2020) 086 [arXiv: 2001.00017] [inSPIRE].

[37] K. Hagiwara, S. Ishihara, R. Szalapski and D. Zeppenfeld, Low-energy effects of new interactions in the electroweak boson sector, Phys. Rev. D 48 (1993) 2182 [INSPIRE].

[38] K. Hagiwara, S. Matsumoto, D. Haidt and C.S. Kim, A Novel approach to confront electroweak data and theory, Z. Phys. C 64 (1994) 559 [Erratum ibid. 68 (1995) 352] [hep-ph/9409380] [INSPIRE].

[39] S. Alam, S. Dawson and R. Szalapski, Low-energy constraints on new physics revisited, Phys. Rev. D 57 (1998) 1577 [hep-ph/9706542] [INSPIRE]. 
[40] H. Mebane, N. Greiner, C. Zhang and S. Willenbrock, Constraints on Electroweak Effective Operators at One Loop, Phys. Rev. D 88 (2013) 015028 [arXiv: 1306.3380] [INSPIRE].

[41] C.-Y. Chen, S. Dawson and C. Zhang, Electroweak Effective Operators and Higgs Physics, Phys. Rev. D 89 (2014) 015016 [arXiv:1311.3107] [InSPIRE].

[42] J. Elias-Miró, J.R. Espinosa, E. Masso and A. Pomarol, Renormalization of dimension-six operators relevant for the Higgs decays $h \rightarrow \gamma \gamma, \gamma Z$, JHEP 08 (2013) 033 [arXiv:1302.5661] [INSPIRE].

[43] J. Elias-Miró, C. Grojean, R.S. Gupta and D. Marzocca, Scaling and tuning of EW and Higgs observables, JHEP 05 (2014) 019 [arXiv:1312.2928] [INSPIRE].

[44] E.E. Jenkins, A.V. Manohar and M. Trott, Renormalization Group Evolution of the Standard Model Dimension Six Operators II: Yukawa Dependence, JHEP 01 (2014) 035 [arXiv:1310.4838] [INSPIRE].

[45] R. Alonso, H.-M. Chang, E.E. Jenkins, A.V. Manohar and B. Shotwell, Renormalization group evolution of dimension-six baryon number violating operators, Phys. Lett. B 734 (2014) 302 [arXiv: 1405.0486] [INSPIRE].

[46] G. Panico, A. Pomarol and M. Riembau, EFT approach to the electron Electric Dipole Moment at the two-loop level, JHEP 04 (2019) 090 [arXiv: 1810.09413] [INSPIRE].

[47] ACME collaboration, Improved limit on the electric dipole moment of the electron, Nature 562 (2018) 355 [INSPIRE].

[48] MEG II collaboration, The design of the MEG II experiment, Eur. Phys. J. C 78 (2018) 380 [arXiv: 1801.04688] [INSPIRE].

[49] P. Baratella, C. Fernandez and A. Pomarol, Renormalization of Higher-Dimensional Operators from On-shell Amplitudes, Nucl. Phys. B (2020) 115155 [arXiv:2005.07129] [INSPIRE].

[50] M. Jiang, T. Ma and J. Shu, Renormalization Group Evolution from On-shell SMEFT, arXiv: 2005.10261 [INSPIRE].

[51] Z. Bern, J. Parra-Martinez and E. Sawyer, Structure of two-loop SMEFT anomalous dimensions via on-shell methods, arXiv:2005.12917 [INSPIRE].

[52] R. Britto, F. Cachazo, B. Feng and E. Witten, Direct proof of tree-level recursion relation in Yang-Mills theory, Phys. Rev. Lett. 94 (2005) 181602 [hep-th/0501052] [INSPIRE]. 